\title{
Measurement of beauty production at HERA using events with muons and jets
}

\section{Journal Article}

\section{Author(s):}

H1 Collaboration; Aktas, A.; Baumgartner, S.; Berger, N.; Erdmann, Wolfram; Grab, Christoph; List, Benno; Mangano, Salvatore; Meer, David; Schöning, André; Weber, R.; et al.

\section{Publication date:}

2005-06

\section{Permanent link:}

https://doi.org/10.3929/ethz-b-000031957

\section{Rights / license:}

Creative Commons Attribution 4.0 International

\section{Originally published in:}

The European Physical Journal C 41(4), https://doi.org/10.1140/epjc/s2005-02267-0 


\title{
Measurement of beauty production at HERA using events with muons and jets
}

\author{
The H1 Collaboration
}

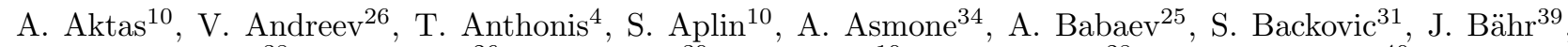
A. Baghdasaryan ${ }^{38}$, P. Baranov ${ }^{26}$, E. Barrelet ${ }^{30}$, W. Bartel ${ }^{10}$, S. Baudrand ${ }^{28}$, S. Baumgartner ${ }^{40}$, J. Becker ${ }^{41}$, M. Beckingham ${ }^{10}$, O. Behnke ${ }^{13}$, O. Behrendt ${ }^{7}$, A. Belousov ${ }^{26}$, Ch. Berger ${ }^{1}$, N. Berger ${ }^{40}$, J.C. Bizot ${ }^{28}$, M.O. Boenig ${ }^{7}$, V. Boudry ${ }^{29}$, J. Bracinik ${ }^{27}$, G. Brandt ${ }^{13}$, V. Brisson ${ }^{28}$, D.P. Brown ${ }^{10}$, D. Bruncko ${ }^{16}$, F.W. Büsser ${ }^{11}$, A. Bunyatyan ${ }^{12,38}$, G. Buschhorn ${ }^{27}$, L. Bystritskaya ${ }^{25}$, A.J. Campbell ${ }^{10}$, S. Caron ${ }^{1}$, F. Cassol-Brunner ${ }^{22}$, K. Cerny ${ }^{33}$, V. Cerny ${ }^{16}$, V. Chekelian ${ }^{27}$, J.G. Contreras ${ }^{23}$, J.A. Coughlan ${ }^{5}$, B.E. Cox ${ }^{21}$, G. Cozzika ${ }^{9}$, J. Cvach ${ }^{32}$, J.B. Dainton ${ }^{18}$, W.D. Dau ${ }^{15}$, K. Daum ${ }^{37,43}$, Y. de Boer ${ }^{25}$, B. Delcourt ${ }^{28}$, R. Demirchyan ${ }^{38}$, A. De Roeck ${ }^{10,45}$, K. Desch ${ }^{11}$, E.A. De Wolf ${ }^{4}$, C. Diaconu ${ }^{22}$, V. Dodonov ${ }^{12}$, A. Dubak ${ }^{31,46}$, G. Eckerlin ${ }^{10}$, V. Efremenko ${ }^{25}$, S. Egli ${ }^{36}$, R. Eichler ${ }^{36}$, F. Eisele ${ }^{13}$, M. Ellerbrock ${ }^{13}$, E. Elsen ${ }^{10}$, W. Erdmann ${ }^{40}$, S. Essenov ${ }^{25}$, P.J.W. Faulkner ${ }^{3}$, L. Favart ${ }^{4}$, A. Fedotov ${ }^{25}$, R. Felst ${ }^{10}$, J. Ferencei ${ }^{10}$, L. Finke ${ }^{11}$, M. Fleischer ${ }^{10}$, P. Fleischmann ${ }^{10}$, Y.H. Fleming ${ }^{10}$, G. Flucke $^{10}$, A. Fomenko ${ }^{26}$, I. Foresti ${ }^{41}$, J. Formánek ${ }^{33}$, G. Franke ${ }^{10}$, G. Frising ${ }^{1}$, T. Frisson ${ }^{29}$, E. Gabathuler ${ }^{18}$, E. Garutti ${ }^{10}$, J. Gayler ${ }^{10}$, R. Gerhards ${ }^{10, \dagger}$, C. Gerlich ${ }^{13}$, S. Ghazaryan ${ }^{38}$, S. Ginzburgskaya ${ }^{25}$, A. Glazov ${ }^{10}$, I. Glushkov ${ }^{39}$, L. Goerlich ${ }^{6}$, M. Goettlich ${ }^{10}$, N. Gogitidze ${ }^{26}$, S. Gorbounov ${ }^{39}$, C. Goyon ${ }^{22}$, C. Grab ${ }^{40}$, T. Greenshaw ${ }^{18}$, M. Gregori ${ }^{19}$, G. Grindhammer ${ }^{27}$, C. Gwilliam ${ }^{21}$, D. Haidt ${ }^{10}$, L. Hajduk ${ }^{6}$, J. Haller ${ }^{13}$, M. Hansson ${ }^{20}$, G. Heinzelmann ${ }^{11}$, R.C.W. Henderson ${ }^{17}$, H. Henschel ${ }^{39}$, O. Henshaw ${ }^{3}$, G. Herrera ${ }^{24}$, I. Herynek ${ }^{32}$, M. Hildebrandt ${ }^{36}$, K.H. Hiller ${ }^{39}$, D. Hoffmann 22 , R. Horisberger ${ }^{36}$, A. Hovhannisyan ${ }^{38}$, M. Ibbotson ${ }^{21}$, M. Ismail ${ }^{21}$, M. Jacquet ${ }^{28}$, L. Janauschek ${ }^{27}$, X. Janssen ${ }^{10}$, V. Jemanov ${ }^{11}$, L. Jönsson ${ }^{20}$, D.P. Johnson ${ }^{4}$, H. Jung ${ }^{20,10}$, M. Kapichine ${ }^{8}$, M. Karlsson ${ }^{20}$, J. Katzy ${ }^{10}$, N. Keller ${ }^{41}$, I.R. Kenyon ${ }^{3}$, C. Kiesling ${ }^{27}$, M. Klein ${ }^{39}$, C. Kleinwort ${ }^{10}$, T. Klimkovich ${ }^{10}$, T. Kluge ${ }^{10}$, G. Knies ${ }^{10}$, A. Knutsson ${ }^{20}$, V. Korbel ${ }^{10}$, P. Kostka ${ }^{39}$, R. Koutouev ${ }^{12}$, K. Krastev ${ }^{35}$, J. Kretzschmar ${ }^{39}$, A. Kropivnitskaya ${ }^{25}$, J. Kroseberg ${ }^{41,47}$, K. Krüger ${ }^{14}$, J. Kückens ${ }^{10}$, M.P.J. Landon ${ }^{19}$, W. Lange ${ }^{39}$, T. Laštovička ${ }^{39,33}$, P. Laycock ${ }^{18}$, A. Lebedev ${ }^{26}$, B. Leißner ${ }^{1}$, V. Lendermann ${ }^{14}$, S. Levonian ${ }^{10}$, L. Lindfeld ${ }^{41}$, K. Lipka ${ }^{39}$, B. List ${ }^{40}$, E. Lobodzinska ${ }^{39,6}$, N. Loktionova ${ }^{26}$, R. Lopez-Fernandez ${ }^{10}$, V. Lubimov ${ }^{25}$, A.-I. Lucaci-Timoce ${ }^{10}$, H. Lueders ${ }^{11}$, D. Lüke ${ }^{70}$, T. Lux $^{11}$, L. Lytkin ${ }^{12}$, A. Makankine ${ }^{8}$, N. Malden ${ }^{21}$, E. Malinovski ${ }^{26}$, S. Mangano ${ }^{40}$, P. Marage ${ }^{4}$, R. Marshall ${ }^{21}$, M. Martisikova ${ }^{10}$, H.-U. Martyn ${ }^{1}$, S.J. Maxfield ${ }^{18}$, D. Meer ${ }^{40}$, A. Mehta ${ }^{18}$, K. Meier ${ }^{14}$, A.B. Meyer ${ }^{11}$, H. Meyer ${ }^{37}$, J. Meyer ${ }^{10}$, S. Mikocki ${ }^{6}$, I. Milcewicz-Mika ${ }^{6}$, D. Milstead ${ }^{18}$, A. Mohamed ${ }^{18}$, F. Moreau ${ }^{29}$, A. Morozov ${ }^{8}$, J.V. Morris ${ }^{5}$, M.U. Mozer ${ }^{13}$, K. Müller ${ }^{41}$, P. Murín ${ }^{16,44}$, K. Nankov ${ }^{35}$, B. Naroska ${ }^{11}$, J. Naumann ${ }^{7}$, Th. Naumann ${ }^{39}$, P.R. Newman ${ }^{3}$, C. Niebuhr ${ }^{10}$, A. Nikiforov ${ }^{27}$, D. Nikitin ${ }^{8}$, G. Nowak ${ }^{6}$, M. Nozicka ${ }^{33}$, R. Oganezov ${ }^{38}$, B. Olivier ${ }^{3}$, J.E. Olsson ${ }^{10}$, S. Osman ${ }^{20}$, D. Ozerov ${ }^{25}$, V. Palichik ${ }^{8}$, T. Papadopoulou ${ }^{10}$, C. Pascaud ${ }^{28}$, G.D. Patel ${ }^{18}$, M. Peez ${ }^{29}$, E. Perez ${ }^{9}$ D. Perez-Astudillo ${ }^{23}$, A. Perieanu ${ }^{10}$, A. Petrukhin ${ }^{25}$, D. Pitzl ${ }^{10}$, R. Plačakytè ${ }^{27}$, B. Portheault ${ }^{28}$, B. Povh ${ }^{12}$, P. Prideaux ${ }^{18}$, N. Raicevic ${ }^{31}$, P. Reimer ${ }^{32}$, A. Rimmer ${ }^{18}$, C. Risler ${ }^{10}$, E. Rizvi ${ }^{3}$, P. Robmann ${ }^{41}$,' B. Roland ${ }^{4}$, R. Roosen ${ }^{4}$, A. Rostovtsev ${ }^{25}$, Z. Rurikova ${ }^{27}$, S. Rusakov ${ }^{26}$, F. Salvaire ${ }^{11}$, D.P.C. Sankey ${ }^{5}$, E. Sauvan ${ }^{22}$, S. Schätzel ${ }^{13}$, F.-P. Schilling ${ }^{10}$, S. Schmidt ${ }^{10}$, S. Schmitt ${ }^{41}$, C. Schmitz ${ }^{41}$, L. Schoeffel ${ }^{9}$, A. Schöning ${ }^{40}$, V. Schröder ${ }^{10}$, H.-C. Schultz-Coulon ${ }^{14}$, C. Schwanenberger ${ }^{10}$, K. Sedlák ${ }^{32}$, F. Sefkow ${ }^{10}$, I. Sheviakov ${ }^{26}$, L.N. Shtarkov ${ }^{26}$, Y. Sirois ${ }^{29}$, T. Sloan ${ }^{17}$, P. Smirnov ${ }^{26}$, Y. Soloviev ${ }^{26}$, D. South ${ }^{10}$, V. Spaskov ${ }^{8}$, A. Specka ${ }^{29}$, B. Stella ${ }^{34}$, J. Stiewe ${ }^{14}$, I. Strauch ${ }^{10}$, U. Straumann ${ }^{41}$, V. Tchoulakov ${ }^{8}$, G. Thompson ${ }^{19}$, P.D. Thompson ${ }^{3}$, F. Tomasz ${ }^{14}$, D. Traynor ${ }^{19}$, P. Truöl ${ }^{41}$, I. Tsakov ${ }^{35}$, G. Tsipolitis ${ }^{10,42}$, I. Tsurin ${ }^{10}$, J. Turnau ${ }^{6}$, E. Tzamariudaki ${ }^{27}$, M. Urban ${ }^{41}$, A. Usik ${ }^{26}$, D. Utkin ${ }^{25}$, S. Valkár ${ }^{33}$, A. Valkárová ${ }^{33}$, C. Vallée ${ }^{22}$, P. Van Mechelen ${ }^{4}$, N. Van Remortel ${ }^{4}$, A. Vargas Trevino ${ }^{7}$, Y. Vazdik ${ }^{26}$, C. Veelken ${ }^{18}$, A. Vest ${ }^{1}$, S. Vinokurova ${ }^{10}$, V. Volchinski ${ }^{38}$, B. Vujicic ${ }^{27}$, K. Wacker ${ }^{7}$, J. Wagner ${ }^{10}$, G. Weber ${ }^{11}$, R. Weber ${ }^{40}$, D. Wegener ${ }^{7}$, C. Werner ${ }^{13}$, N. Werner ${ }^{41}$, M. Wessels ${ }^{10}$, B. Wessling ${ }^{10}$, C. Wigmore ${ }^{3}$, G.-G. Winter ${ }^{10}$, Ch. Wissing ${ }^{7}$, R. Wolf ${ }^{13}$, E. Wünsch ${ }^{10}$, S. Xella ${ }^{41}$, W. Yan ${ }^{10}$, V. Yeganov ${ }^{38}$, J. Žáček ${ }^{33}$, J. Zálešák ${ }^{32}$, Z. Zhang ${ }^{28}$, A. Zhelezov ${ }^{25}$, A. Zhokin ${ }^{25}$, J. Zimmermann ${ }^{27}$, H. Zohrabyan ${ }^{38}$, F. Zomer ${ }^{28}$

1 I. Physikalisches Institut der RWTH, Aachen, Germany ${ }^{\mathrm{a}}$

2 III. Physikalisches Institut der RWTH, Aachen, Germany ${ }^{a}$

3 School of Physics and Astronomy, University of Birmingham, Birmingham, $\mathrm{UK}^{\mathrm{b}}$

4 Inter-University Institute for High Energies ULB-VUB, Brussels; Universiteit Antwerpen, Antwerpen; Belgium ${ }^{c}$

5 Rutherford Appleton Laboratory, Chilton, Didcot, $\mathrm{UK}^{\mathrm{b}}$

6 Institute for Nuclear Physics, Cracow, Poland ${ }^{\mathrm{d}}$ 
7 Institut für Physik, Universität Dortmund, Dortmund, Germany ${ }^{\mathrm{a}}$

8 Joint Institute for Nuclear Research, Dubna, Russia

9 CEA, DSM/DAPNIA, CE-Saclay, Gif-sur-Yvette, France

10 DESY, Hamburg, Germany

11 Institut für Experimentalphysik, Universität Hamburg, Hamburg, Germany ${ }^{a}$

12 Max-Planck-Institut für Kernphysik, Heidelberg, Germany

13 Physikalisches Institut, Universität Heidelberg, Heidelberg, Germany ${ }^{a}$

14 Kirchhoff-Institut für Physik, Universität Heidelberg, Heidelberg, Germany ${ }^{\mathrm{a}}$

15 Institut für experimentelle und Angewandte Physik, Universität Kiel, Kiel, Germany

16 Institute of Experimental Physics, Slovak Academy of Sciences, Košice, Slovak Republic ${ }^{\mathrm{f}}$

17 Department of Physics, University of Lancaster, Lancaster, UK ${ }^{\mathrm{b}}$

18 Department of Physics, University of Liverpool, Liverpool, UK ${ }^{\mathrm{b}}$

19 Queen Mary and Westfield College, London, UK ${ }^{\mathrm{b}}$

20 Physics Department, University of Lund, Lund, Sweden ${ }^{\mathrm{g}}$

21 Physics Department, University of Manchester, Manchester, UK ${ }^{\mathrm{b}}$

22 CPPM, CNRS/IN2P3 - Univ Mediterranee, Marseille - France

23 Departamento de Fisica Aplicada, CINVESTAV, Mérida, Yucatán, México ${ }^{\mathrm{k}}$

24 Departamento de Fisica, CINVESTAV, México $^{\mathrm{k}}$

25 Institute for Theoretical and Experimental Physics, Moscow, Russia ${ }^{1}$

${ }^{26}$ Lebedev Physical Institute, Moscow, Russia ${ }^{\text {e }}$

27 Max-Planck-Institut für Physik, München, Germany

28 LAL, Université de Paris-Sud, IN2P3-CNRS, Orsay, France

29 LLR, Ecole Polytechnique, IN2P3-CNRS, Palaiseau, France

30 LPNHE, Universités Paris VI and VII, IN2P3-CNRS, Paris, France

31 Faculty of Science, University of Montenegro, Podgorica, Serbia and Montenegro

32 Institute of Physics, Academy of Sciences of the Czech Republic, Praha, Czech Republic ${ }^{\mathrm{e}, \mathrm{i}}$

33 Faculty of Mathematics and Physics, Charles University, Praha, Czech Republic ${ }^{\mathrm{e}, \mathrm{i}}$

34 Dipartimento di Fisica Università di Roma Tre and INFN Roma 3, Roma, Italy

35 Institute for Nuclear Research and Nuclear Energy, Sofia,Bulgaria

36 Paul Scherrer Institut, Villingen, Switzerland

37 Fachbereich C, Universität Wuppertal, Wuppertal, Germany

38 Yerevan Physics Institute, Yerevan, Armenia

39 DESY, Zeuthen, Germany

40 Institut für Teilchenphysik, ETH, Zürich, Switzerland ${ }^{\mathrm{j}}$

41 Physik-Institut der Universität Zürich, Zürich, Switzerland ${ }^{\mathrm{j}}$

$\dagger$ Deceased

Received: 7 February 2005 / Revised version: 26 April 2005 /

Published online: 31 May 2005 - (c) Springer-Verlag / Società Italiana di Fisica 2005

\begin{abstract}
A measurement of the beauty production cross section in ep collisions at a centre-of-mass energy of $319 \mathrm{GeV}$ is presented. The data were collected with the H1 detector at the HERA collider in the years 1999-2000. Events are selected by requiring the presence of jets and muons in the final state. Both the long lifetime and the large mass of $b$-flavoured hadrons are exploited to identify events containing beauty quarks. Differential cross sections are measured in photoproduction, with photon virtualities $Q^{2}<1 \mathrm{GeV}^{2}$, and in deep inelastic scattering, where $2<Q^{2}<100 \mathrm{GeV}^{2}$. The results are compared with perturbative QCD calculations to leading and next-to-leading order. The predictions are found to be somewhat lower than the data.
\end{abstract}

\footnotetext{
${ }_{42}$ Also at Physics Department, National Technical University, Zografou Campus, GR-15773 Athens, Greece

43 Also at Rechenzentrum, Universität Wuppertal, Wuppertal, Germany

44 Also at University of P.J. Šafárik, Košice, Slovak Republic

45 Also at CERN, Geneva, Switzerland

${ }^{46}$ Also at Max-Planck-Institut für Physik, München, Germany

47 Now at UC Santa Cruz, California, USA

a Supported by the Bundesministerium für Bildung und Forschung, FRG, under contract numbers 05 H1 1GUA /1, 05 H1 1PAA / 1, 05 H1 1PAB /9, 05 H1 1PEA /6, 05 H1 1VHA
}

\footnotetext{
$/ 7$ and $05 \mathrm{H} 1$ 1VHB /5

b Supported by the UK Particle Physics and Astronomy Research Council, and formerly by the UK Science and Engineering Research Council

${ }^{\text {c }}$ Supported by FNRS-FWO-Vlaanderen, IISN-IIKW and IWT and by Interuniversity Attraction Poles Programme, Belgian Science Policy

${ }^{d}$ Partially Supported by the Polish State Committee for Scientific Research, SPUB/DESY/P003/DZ 118/2003/2005

e Supported by the Deutsche Forschungsgemeinschaft

${ }^{f}$ Supported by VEGA SR grant no. 2/4067/ 24
} 


\section{Introduction}

A measurement is presented of open beauty production $e p \rightarrow e b \bar{b} X$ in $e p$ collisions with the $\mathrm{H} 1$ detector at HERA. The measurement spans the kinematic range from the domain of photoproduction, in which the exchanged photon is quasi-real $\left(Q^{2} \sim 0\right)$, to the region of electroproduction, or deep inelastic scattering (DIS), with photon virtualities $2<Q^{2}<100 \mathrm{GeV}^{2}$. For beauty production, calculations in perturbative quantum chromodynamics (pQCD) are expected to give reliable predictions, as the mass $m_{b}$ of the $b$ quark $\left(m_{b} \sim 5 \mathrm{GeV}\right)$ provides a hard scale. With the phase space covered in this analysis the interplay of the hard scales $m_{b}, Q^{2}$ and the transverse momenta of the $b$ quarks can be probed.

First measurements of the beauty cross section at HERA $[1,2]$ were higher than pQCD predictions calculated at next-to-leading order (NLO). Similar observations were made in hadron-hadron collisions [3] and also in twophoton interactions [4]. Recent beauty production measurements from the H1 [5] and ZEUS Collaborations [6] are in better agreement with QCD predictions or again somewhat higher [7].

In this paper, photoproduction events with at least two jets $(j j)$ and a muon $(\mu)$ in the final state are used to measure the beauty cross section for $e p \rightarrow e b \bar{b} X \rightarrow e j j \mu X^{\prime}$. In deep inelastic scattering, the process $e p \rightarrow e b \bar{b} X \rightarrow e j \mu X^{\prime}$ is measured with at least one jet and a muon in the final state. For the first time at HERA, two distinct features of $B$-hadrons are exploited simultaneously to discriminate events containing beauty from those with only charm or light quarks: the large mass and the long lifetime. The $B$-hadron mass leads to a broad distribution of the transverse momentum $p_{t}^{\text {rel }}$ of decay muons relative to the beauty quark jet direction. The $B$-hadron lifetime is reflected in the large impact parameters $\delta \sim 200 \mu \mathrm{m}$ of decay muon tracks relative to the primary vertex. The precision measurement of muon track impact parameters is made possible by the H1 Central Silicon Track detector [8]. The fractions of $b$ quark events in the data samples are determined from a fit to the two-dimensional distribution of $p_{t}^{\text {rel }}$ and $\delta$.

This paper is organized as follows. In Sect. 2, an introduction to the physics of beauty production in $e p$ collisions is given. The relevant features of the H1 detector are described in Sect. 3. Section 4 describes the event selection. The Monte Carlo simulations and NLO QCD calculations are presented in Sects. 5 and 6. Comparisons of the data samples with the Monte Carlo simulations are shown in Sect. 7. The fit procedure used to determine the $b$-fraction and the systematic errors of the measurement

\footnotetext{
g Supported by the Swedish Natural Science Research Council

i Supported by the Ministry of Education of the Czech Republic under the projects INGO-LA116/2000 and LN00A006, by GAUK grant no $173 / 2000$

j Supported by the Swiss National Science Foundation

k Supported by CONACYT, México, grant 400073-F

1 Partially Supported by Russian Foundation for Basic Research, grant no. 00-15-96584
}

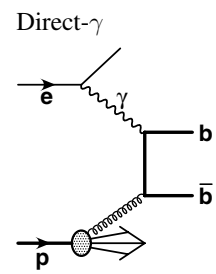

a $\gamma g$-Fusion

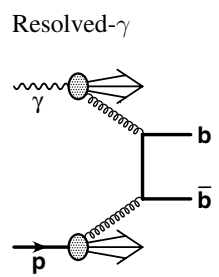

b Hadron-like c $b$-Excitation

d $b$-Excitation

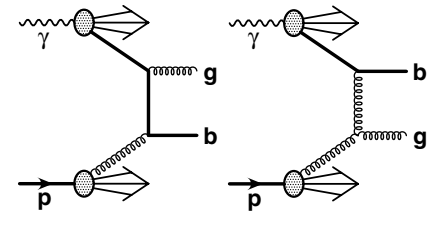

Fig. 1a-d. Beauty production processes in leading order pQCD

are explained in Sects. 8 and 9. Finally, the results are presented in Sect. 10 and conclusions are drawn in Sect. 11.

\section{Heavy quark production in $e p$ collisions}

In pQCD, at leading order, two distinct classes of processes contribute to the production of beauty quarks in $e p$ collisions at HERA. In direct-photon processes (Fig. 1a), the photon emitted from the positron enters the hard process $\gamma g \rightarrow b \bar{b}$ directly. In resolved-photon processes (Figs. $1 \mathrm{~b}$ to $\mathrm{d}$ ), the photon fluctuates into a hadronic state before the hard interaction and acts as a source of partons, one of which takes part in the hard interaction. Resolved photon processes are expected to contribute significantly in the photoproduction region, in which the photon is quasi-real, and to be suppressed towards higher $Q^{2}$.

For the kinematic range covered in this analysis, the majority of events are in the region $p_{t, b} \gtrsim m_{b}$, where $p_{t, b}$ is the momentum of the outgoing $b$ quark transverse to the photon-proton axis in the photon-proton centreof-mass frame. In this region, NLO calculations in the massive scheme $[9,10]$ are expected to give reliable results. In this scheme, $u, d$ and $s$ are the only active flavours in the proton and the photon, and charm and beauty are produced dynamically in the hard scattering. At large transverse momenta $p_{t, b} \gg m_{b}$ or large $Q^{2} \gg\left(2 m_{b}\right)^{2}$, the massive scheme becomes unreliable due to large terms in the perturbation series of the form $\alpha_{s} \ln \left(p_{t, b}^{2} / m_{b}^{2}\right)$ or $\alpha_{s} \ln \left(Q^{2} / m_{b}^{2}\right)$. In this kinematic range, the massless scheme [11] can be used, in which charm and beauty are treated as active flavours in both the proton and the photon, in addition to $u, d$ and $s$. In this scheme, so-called excitation processes occur in which the beauty quark is a constituent of the resolved photon (sketched in Figs. 1c and d) or of the proton.

In this analysis the measurements are compared with NLO calculations in the massive scheme for both photoproduction [9] and DIS [10]. NLO calculations in the massless scheme are not yet available for the exclusive final state considered in this measurement. The data are also compared with the predictions of the Monte Carlo simulations PYTHIA [12], RAPGAP [13] and CASCADE [14]. In the Monte Carlo simulations leading order matrix elements are implemented and higher orders are approximated using parton showers radiated from the initial and final state partons. PYTHIA and RAPGAP use the DGLAP [15] parton evolution equations, while CASCADE 
contains an implementation of the CCFM [16] evolution equation. In CASCADE the direct process $\gamma g \rightarrow b \bar{b}$ is implemented using off-shell matrix elements convoluted with $k_{t}$-unintegrated parton distributions in the proton.

\section{Detector description}

The $\mathrm{H} 1$ detector is described in detail in [17] and only the components most relevant for this analysis are briefly discussed here. A right handed coordinate system is employed that has its $z$-axis pointing in the proton beam, or forward, direction and $x(y)$ pointing in the horizontal (vertical) direction. Charged particles are measured in the Central Tracking Detector (CTD) which covers the range in pseudo-rapidity between $-1.74<\eta<1.74^{1}$. The CTD comprises two large cylindrical Central Jet Chambers (CJCs) and two z-chambers arranged concentrically around the beam-line within a solenoidal magnetic field of $1.15 \mathrm{~T}$. The CTD also provides trigger information which is based on track segments in the $r-\phi$ plane measured in the CJCs and the $z$-position of the interaction vertex obtained from a double layer of multi-wire proportional chambers.

The CTD tracks are linked with hits in the Central Silicon Track detector (CST) [8], which consists of two 36 $\mathrm{cm}$ long concentric cylindrical layers of silicon strip detectors, surrounding the beam pipe at radii of $57.5 \mathrm{~mm}$ and $97 \mathrm{~mm}$ from the beam axis. The CST covers a pseudorapidity range of $-1.3<\eta<1.3$ for tracks passing through both layers. The double-sided silicon detectors provide resolutions of $12 \mu \mathrm{m}$ in $r$ - $\phi$ and $25 \mu \mathrm{m}$ in $z$. Average hit efficiencies are $97 \%(92 \%)$ in $r-\phi(z)$. For a CTD track with CST $r-\phi$ hits in both layers, the transverse distance of closest approach $d c a$ of the track to the nominal vertex in $x$ - $y$ can be measured with a resolution of $\sigma_{d c a} \approx 33 \mu \mathrm{m} \oplus 90 \mu \mathrm{m} / p_{t}[\mathrm{GeV}]$, where the first term represents the intrinsic resolution (including alignment uncertainties) and the second term is the contribution from multiple scattering in the beam pipe and the CST; $p_{t}$ is the transverse momentum of the track.

The track detectors are surrounded in the forward and central directions, $-1.5<\eta<3.4$, by a fine grained Liquid Argon calorimeter (LAr) and in the backward region, $-4.0<\eta<-1.4$, by a lead-scintillating fibre calorimeter SpaCal [18] with electromagnetic and hadronic sections. The SpaCal is used primarily in this analysis to detect the scattered electron in DIS events and to select photoproduction events, in which case the scattered electron is not detected. The calorimeters are surrounded by the solenoidal magnet and the iron return yoke which is instrumented with 16 layers of limited streamer tubes in the range $-2.5<\eta<3.4$, allowing the identification of muon tracks.

The ep luminosity is determined by measuring the QED bremsstrahlung ( $e p \rightarrow e p \gamma$ ) event rate by detecting

\footnotetext{
1 The pseudo-rapidity $\eta$ corresponding to a polar angle $\theta$ is given by $\eta=-\ln \tan (\theta / 2)$.
}

the radiated photon in a calorimeter located at $z=-103$ m.

\section{Event selection and reconstruction}

The data were recorded in the years 1999 and 2000 and correspond to an integrated luminosity of $\sim 50 \mathrm{pb}^{-1}$. During this time HERA was operated with positrons of 27.6 $\mathrm{GeV}$ and protons of $920 \mathrm{GeV}$ energy. The events were triggered by requiring that there be signals from the central drift chambers and the multi-wire proportional chambers in coincidence with signals from the scattered positron in the backward calorimeter (DIS sample) or with signals from the instrumented iron (photoproduction).

At least one muon is required with a transverse momentum $p_{t}^{\mu}>2.5 \mathrm{GeV}$. Muons are identified as track segments in the barrel part of the instrumented iron. The iron track segments must be well matched to a track reconstructed in the CTD. At least two CST $r$ - $\phi$-hits have to be associated with the muon track and it is required that the combined CTD-CST $r$ - $\phi$-track fit probability exceeds $10 \%$. The muon momentum is reconstructed using the CTD-CST track information. The CST hit requirements for the muon track restrict the allowed range of $e p$ interactions along the $z$-axis to $\left|z_{v t x}\right| \leq 20 \mathrm{~cm}$.

Jets are reconstructed using the inclusive $k_{t}$ algorithm $[19,20]$ in the $p_{t}$ recombination scheme (see [21]), giving massless jets. The algorithm, with a distance parameter in the $\eta$ - $\phi$ plane of 1 , is applied to all hadronic final state particles, which are reconstructed using a combination of tracks and calorimeter energy deposits [22]. The muon, as measured in the CTD and CST, is one of the particles which is input to the jet algorithm. The muon track is required to be associated with one of the selected jets by the jet algorithm.

For the selection of photoproduction events it is required that there be no scattered electron candidate in the backward or central calorimeter. This restricts the accepted range of negative four-momentum transfer squared to $Q^{2}<1 \mathrm{GeV}^{2}$ with a mean of about $0.07 \mathrm{GeV}^{2}$. For the photoproduction sample, a cut on the inelasticity $0.2<y<0.8$ is applied, where $y$ is reconstructed using the relation $y=\sum_{h}\left(E-p_{z}\right) / 2 E_{e}$ [23]. Here, $E$ and $p_{z}$ are the energies and $z$-components of the momenta of the hadronic final state particles, $h$, and $E_{e}$ is the positron beam energy. The final photoproduction event sample consists of 1745 events. The number of events containing more than one muon candidate is less than $1 \%$. The jet algorithm is applied in the laboratory frame and at least two jets are required with transverse momenta $p_{t}^{\text {jet }_{1(2)}}>7(6) \mathrm{GeV}$ in the pseudo-rapidity range $\left|\eta^{\text {jet }}\right|<2.5$. The fraction of the photon energy entering the hard interaction is estimated using the observable

$$
x_{\gamma}^{\mathrm{obs}}=\frac{\sum_{\mathrm{Jet}_{1}}\left(E-p_{z}\right)+\sum_{\mathrm{Jet}_{2}}\left(E-p_{z}\right)}{\sum_{h}\left(E-p_{z}\right)},
$$

where the sums in the numerator run over the particles associated with the two jets and that in the denominator over all detected hadronic final state particles. For 
Table 1. Selection cuts for the photoproduction and DIS data samples and the number of selected events

\begin{tabular}{lll}
\hline & Photoproduction & DIS \\
\hline$Q^{2}\left[\mathrm{GeV}^{2}\right]$ & $<1$ & $2 \ldots 100$ \\
$y$ & $0.2 \ldots 0.8$ & $0.1 \ldots 0.7$ \\
\hline Frame & laboratory & Breit \\
$\#$ jets & $\geq 2$ & $\geq 1$ \\
$p_{t}^{\text {jet }}[\mathrm{GeV}]$ & $>7(6)$ & $>6$ \\
$\left|\eta_{\text {lab }}^{\text {jet }}\right|$ & $<2.5$ & $<2.5$ \\
\hline$\mu$ Iron link probability & $>10 \%$ & $>5 \%$ \\
$\mu$ CST link probability & $>10 \%$ & $>10 \%$ \\
$\#$ CST hits & $\geq 2$ & $\geq 2$ \\
$\eta^{\mu}$ & $-0.55 \ldots 1.1$ & $-0.75 \ldots 1.15$ \\
$p_{t}^{\mu}[\mathrm{GeV}]$ & $>2.5$ & $>2.5$ \\
\hline$\#$ events & 1745 & 776 \\
\hline
\end{tabular}

the direct process (Fig. 1a), $x_{\gamma}^{\text {obs }}$ approaches unity, as the hadronic final state consists of only the two hard jets and the proton remnant in the forward region which contributes little to $\sum_{h}\left(E-p_{z}\right)$. In resolved processes $x_{\gamma}^{\text {obs }}$ can be small.

DIS events are selected by requiring a scattered positron signal with an energy of at least $8 \mathrm{GeV}$ in the SpaCal. To suppress photoproduction background and to reduce the fraction of events with significant initial state QED radiation, events are rejected if $\sum\left(E-p_{z}\right)<45 \mathrm{GeV}$. Here, $E-p_{z}$ is summed over all final state particles including the scattered positron. The kinematic variables $Q^{2}$ and $y$ are reconstructed using the $e \Sigma$ method [24], which combines the hadronic final state and the positron measurements. The scaling variable $x$ is subsequently calculated using the relation $x=Q^{2} / y s$, where $s$ is the $e p$ centre-of-mass energy squared. Events are selected in the range $2<Q^{2}<100 \mathrm{GeV}^{2}$ and $0.1<y<0.7$. The jet algorithm is applied in the Breit frame [25] and at least one jet with transverse momentum $p_{t, \text { jet }}^{\text {Breit }}>6 \mathrm{GeV}$ is required, with which the muon must be associated. The final DIS event sample consists of 776 events.

Table 1 summarises the selection cuts for the two samples. The selection cuts for the photoproduction sample are somewhat tighter, due to the muon trigger acceptance and to suppress background events.

\section{Monte Carlo simulations}

The Monte Carlo generators PYTHIA [12] and RAPGAP [13] are used for the description of the signal and background distributions, the determination of efficiencies and acceptances and for systematic studies. The track resolutions were adjusted to describe the data. In addition, systematic cross checks and estimates of model dependence are performed using the CASCADE generator [14]. The measured beauty production cross sections are also compared with the predictions of these three generators.
The basic parameter choices for the various $\mathrm{PQCD}$ programs are summarised in Table 2.

All three Monte Carlo generators are used to produce large samples of beauty and charm events with decays into muons $e p \rightarrow e b \bar{b} X \rightarrow \mu X^{\prime}$ or $e p \rightarrow e c \bar{c} X \rightarrow \mu X^{\prime}$. The Peterson fragmentation function [26] is used for the hadronisation of the heavy quarks. For systematic cross checks, samples using the Lund string fragmentation model [27] are generated. Each of these Monte Carlo samples corresponds to at least forty times the luminosity of the data. In addition, PYTHIA and RAPGAP event samples for light quark $(u d s), c$ and $b$ events without muon requirements are generated with six times the luminosity of the data. The latter samples are used for the simulation of the background due to hadrons misidentified as muons and decays of light hadrons into muons. All generated events are passed through a detailed simulation of the detector response based on the GEANT program [28] and reconstructed using the same reconstruction software as used for the data.

For the measurements in photoproduction, PYTHIA is used in an inclusive mode in which direct and resolved events are generated using massless matrix elements for all quark flavours $(\operatorname{MSTP}(14)=30[12])$. Beauty (charm) events are separated from light quark events by requiring that there be at least one beauty (charm) quark in the list of outgoing hard partons. Approximately $35 \%$ of the PYTHIA beauty cross section in the measured range is due to resolved photon processes and these are dominated by the flavour excitation component. For the measurements in the DIS region, RAPGAP is used to generate the direct production process in the massive mode (IPRO $=14$ [13]). RAPGAP is interfaced with the program HERACLES [29] which simulates QED initial and final state radiation. Additional event samples are generated using the Monte Carlo generator CASCADE [14]. Comparisons of the measurements with the predictions of CASCADE are made using version 1.2 of the program and the J2003 parton density distributions [30-32].

All generators use the JETSET part of the PYTHIA [12] program to simulate the hadronisation and decay processes. The branching ratios for the direct semileptonic decays $b \rightarrow \mu X$ and for the indirect decays into muons via charm, anticharm, $\tau$ and $J / \Psi$ decays are in agreement with the world average values [35]. The total branching ratio for beauty decays into muons is $21 \%$ [35]. The decay lifetimes of the beauty and charm hadrons are set to the values reported in [35]. The muon momentum spectrum in the rest frame of the decaying $b$-flavoured hadrons, as modeled by JETSET, is in agreement with the spectrum measured at $e^{+} e^{-}$colliders $[36,37]$.

\section{NLO QCD calculations}

The NLO pQCD calculations are performed in the massive scheme using the program FMNR [9] in the photoproduction regime and the program HVQDIS [10] for the DIS case. Both programs provide weighted parton level events with two or three outgoing partons, i.e. a $b$ quark, 
Table 2. Parameters used in the leading order Monte Carlo simulations and the NLO programs. Here $\mu_{r}$ and $\mu_{f}$ denote the renormalisation and factorisation scales, $m_{q}$ the heavy quark masses, $p_{t q \bar{q}}^{2}$ the average of the squared transverse momenta of the two heavy quarks, $\hat{s}$ and $Q_{t}^{2}$ the heavy quark system centre-of-mass energy squared and transverse momentum squared, respectively, and $\epsilon_{q}$ the Peterson fragmentation parameters

\begin{tabular}{|c|c|c|c|c|c|}
\hline & PYTHIA & RAPGAP & CASCADE & FMNR & HVQDIS \\
\hline Version & 6.1 & 2.8 & $1.00 / 09 ; 1.2$ & & 1.4 \\
\hline Proton PDF & CTEQ5L [33] & CTEQ5L & $\begin{array}{l}\text { JS2001 [14] } \\
\text { J2003 [30-32] }\end{array}$ & CTEQ5M [33] & CTEQ5F4 [33] \\
\hline Photon PDF & GRV-G LO [34] & & & GRV-G HO [34] & \\
\hline$\Lambda_{Q C D}^{(4)}[\mathrm{GeV}]$ & 0.192 & 0.192 & 0.2 & 0.326 & 0.309 \\
\hline Renorm. scale $\mu_{r}^{2}$ & $m_{q}^{2}+p_{t q \bar{q}}^{2}$ & $Q^{2}+p_{t q \bar{q}}^{2}$ & $\hat{s}+p_{t q \bar{q}}^{2}$ & $m_{b}^{2}+p_{t b \bar{b}}^{2}$ & $m_{b}^{2}+p_{t b \bar{b}}^{2}$ \\
\hline Factor. scale $\mu_{f}^{2}$ & $m_{q}^{2}+p_{t q \bar{q}}^{2}$ & $Q^{2}+p_{t q \bar{q}}^{2}$ & $\hat{s}+Q_{t}^{2}$ & $m_{b}^{2}+p_{t b \bar{b}}^{2}$ & $m_{b}^{2}+p_{t b \bar{b}}^{2}$ \\
\hline$m_{b}[\mathrm{GeV}]$ & 4.75 & 4.75 & 4.75 & 4.75 & 4.75 \\
\hline$m_{c}[\mathrm{GeV}]$ & 1.5 & 1.5 & 1.5 & & \\
\hline Peterson $\epsilon_{b}$ & 0.0069 & 0.0069 & 0.0069 & 0.0033 & 0.0033 \\
\hline Peterson $\epsilon_{c}$ & 0.058 & 0.058 & 0.058 & & \\
\hline
\end{tabular}

a $\bar{b}$ quark and possibly an additional light parton. The calculations are performed in the $\overline{\mathrm{MS}}$-scheme using the parameters given in Table 2 .

The $b$ quark is 'hadronised' into a $b$-flavoured hadron by rescaling the three-momentum of the quark using the Peterson fragmentation function [26] with the parameter $\epsilon_{b}=0.0033$ [38]. The programs are extended to include the decay of the $b$-flavoured hadron into a final state with a muon. The muon decay spectrum is taken from JETSET [12] and includes direct and indirect decays of $b$-flavoured hadrons into muons. Parton level jets are reconstructed by applying the $k_{t}$ jet algorithm to the outgoing partons.

Corrections to the hadron level are calculated using the PYTHIA and RAPGAP Monte Carlo event generators. PYTHIA and RAPGAP parton level jets are reconstructed from the generated quarks and gluons after the parton showering step. At the hadron level, jets are reconstructed by applying the jet algorithm to all final state particles, after the decay of the beauty or charm hadrons. The jet and the muon selection cuts are applied to the generator samples. In each kinematic bin of the measurement, the ratio of the PYTHIA or RAPGAP hadron level and parton level cross sections is calculated and applied as a correction factor to the NLO calculation. The parton to hadron level corrections range typically from $-30 \%$ to $+5 \%$ in both photoproduction and DIS. The corrections are negative at small muon and jet transverse momenta and positive at the largest transverse momenta. The corrections obtained using CASCADE are consistent with the values from PYTHIA (photoproduction) and RAPGAP (DIS).

The theoretical uncertainties of the NLO calculations are estimated in the following way: The $b$ quark mass and the renormalisation and factorisation scales are varied simultaneously from $m_{b}=4.5 \mathrm{GeV}$ and $\mu_{r}=\mu_{f}=m_{T} / 2$ to $m_{b}=5 \mathrm{GeV}$ and $\mu_{r}=\mu_{f}=2 m_{T}$, where $m_{T}=$ $\sqrt{m_{b}^{2}+p_{t b \bar{b}}^{2}}$, and $p_{t b \bar{b}}^{2}$ is the average of the squared transverse momenta of the two $b$ quarks. This leads to a maximum change of the cross section of typically $25 \%$ in photoproduction (FMNR) and 15-20\% in DIS (HVQDIS). The cross section variation when using other proton structure functions such as MRSG or MRST1 [39] is less than $8 \%$ in all regions of the measurement. The uncertainty due to variations of the fragmentation parameter $\epsilon_{b}$ by $25 \%$ is below $3 \%$. These cross section variations are added in quadrature to estimate the total systematic uncertainty of the NLO predictions for each bin of the measurement.

\section{Comparison of the data with Monte Carlo simulations}

Detailed comparisons are performed of the data with the Monte Carlo simulations. The good agreement of the PYTHIA simulation with the photoproduction data is illustrated in Fig. 2. The distributions of the muon transverse momentum $p_{t}^{\mu}$, the pseudo-rapidity $\eta^{\mu}$, the jet transverse momentum $p_{t}^{\text {jet }_{1(2)}}$ and $x_{\gamma}^{\text {obs }}$ are shown. The data are compared with the sum of the contributions from beauty, charm and light quark events, the relative fractions of which are taken from the two-dimensional fit discussed in Sect. 8. The number of events in the simulation is normalised to that of the data. It is observed that the shapes of the distributions are rather similar for the beauty, charm and light quark events except for the muon transverse momentum, where the spectrum is harder for beauty than for the other components. In Fig. 3, the distributions for the DIS sample are shown. The photon virtuality $Q^{2}$, the inelasticity $y$, Bjorken- $x$, the muon transverse momentum $p_{t}^{\mu}$ and the transverse momentum $p_{t, \mathrm{jet}}^{\text {Breit }}$ 


\section{Photoproduction}
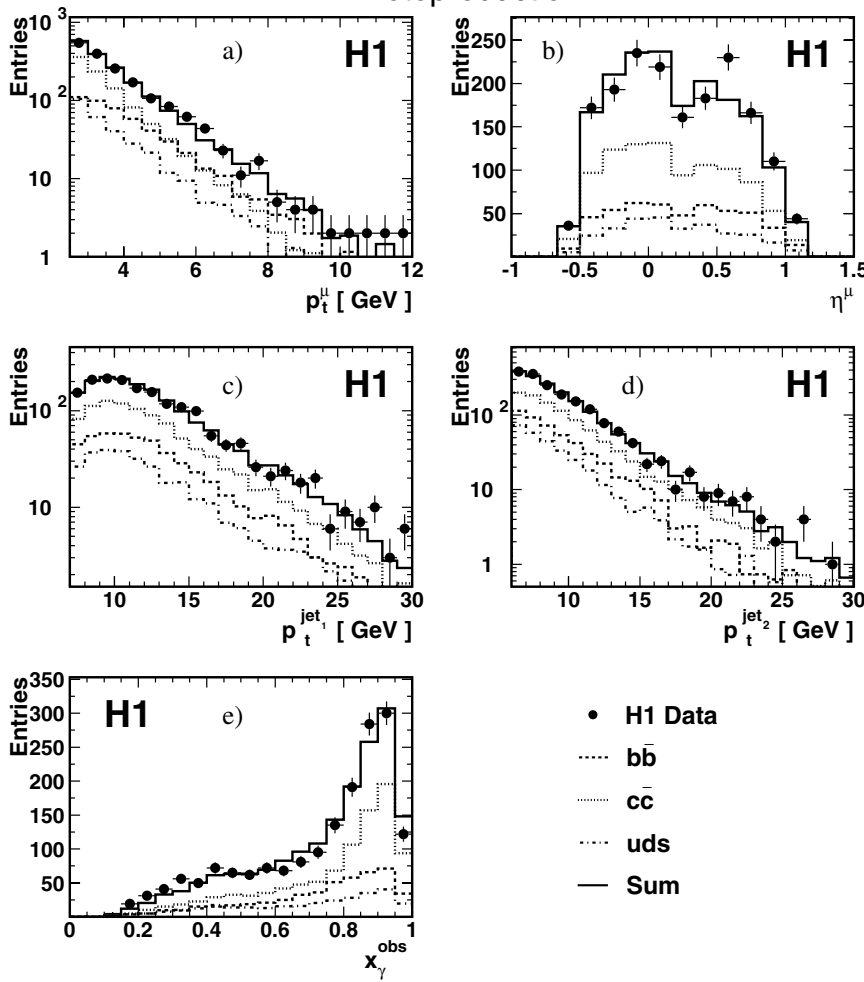

Fig. 2. Distributions in photoproduction of a the muon transverse momentum $p_{t}^{\mu}$, $\mathbf{b}$ the pseudo-rapidity of the muon $\eta^{\mu}$, $\mathbf{c}$ and $\mathbf{d}$ the transverse momenta $p_{t}^{\text {jet }_{1(2)}}$ of the highest and the second-highest $p_{t}$ jets, respectively and e the observable $x_{\gamma}^{\text {obs }}$. Included in the figure are the estimated contributions of events arising from $b$ quarks (dashed line), $c$ quarks (dotted line) and light quarks (dash-dotted line). The shapes of the distributions from the different sources are taken from the PYTHIA Monte Carlo simulation and their relative fractions are determined from a fit to the two-dimensional data distribution of $p_{t}^{\text {rel }}$ and $\delta$ (see text)

of the selected jet in the Breit frame are well described by the RAPGAP Monte Carlo simulation. The CASCADE Monte Carlo simulation also provides a good description of the data in both photoproduction and DIS (not shown).

\section{Determination of beauty and background contributions}

The signed impact parameter $\delta$ of the muon track and the transverse momentum $p_{t}^{\text {rel }}$ of the muon track relative to the axis of the associated jet are used to determine the fraction of beauty events in the data. For each muon candidate, $\delta$ is calculated in the plane transverse to the beam axis. The magnitude of $\delta$ is given by the $d c a$ of the track to the primary vertex. The sign is defined as positive if the angle between the jet axis and the line joining the primary vertex to the point of closest approach of the track is less than $90^{\circ}$, and is defined as negative otherwise. Figures $4 \mathrm{a}$ and $5 \mathrm{a}$ show the distributions of $\delta$ for
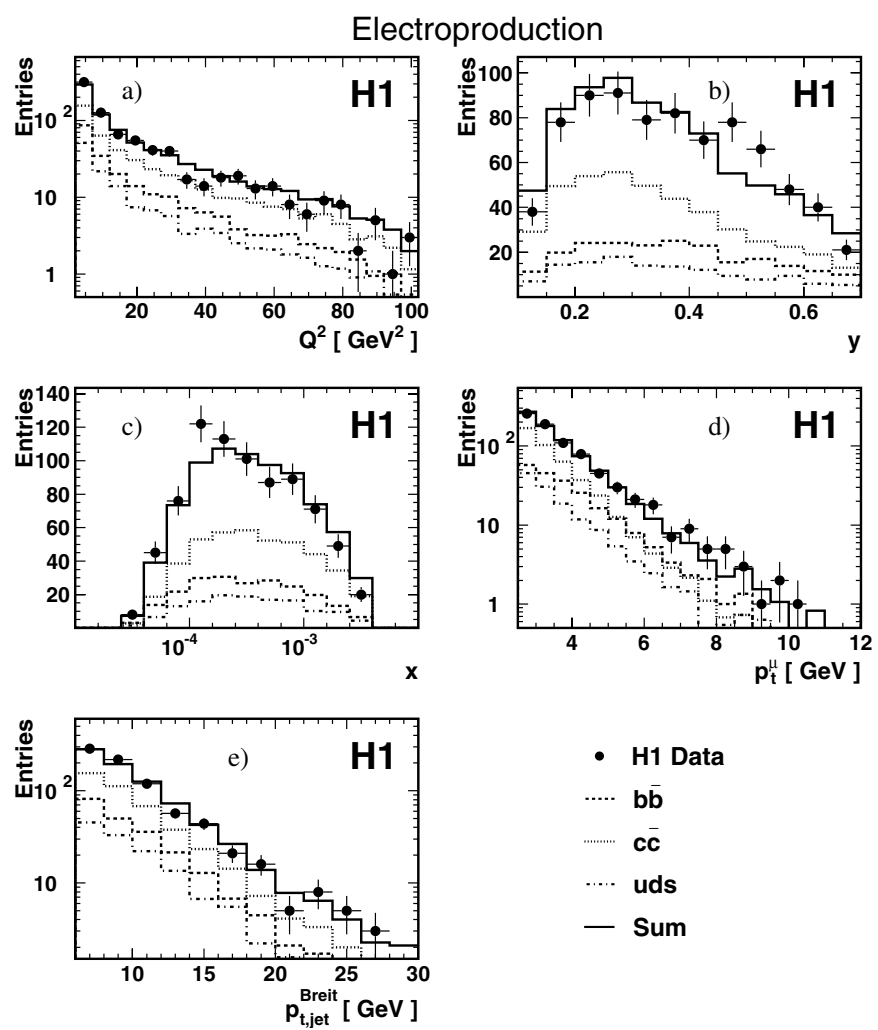

Fig. 3. Distributions in electroproduction of a the photon virtuality $Q^{2}, \mathbf{b}$ the inelasticity $y, \mathbf{c}$ Bjorken $\mathrm{x}, \mathbf{d}$ the muon transverse momentum and e the transverse momentum $p_{t, \text { jet }}^{\text {Breit }}$ of the selected jet in the Breit frame. Included in the figure are the estimated contributions of events arising from $b$ quarks (dashed line), $c$ quarks (dotted line) and light quarks (dashdotted line). The shapes of the distributions from the different sources are taken from the RAPGAP Monte Carlo simulation and their relative fractions are determined from a fit to the two-dimensional data distribution of $p_{t}^{\text {rel }}$ and $\delta$ (see text)

the photoproduction and DIS samples, respectively. The decay of long-lived particles mainly leads to positive impact parameters, whereas particles produced at the vertex yield a symmetric distribution centered at zero with finite width due to the track and primary vertex resolutions.

The transverse beam interaction region at HERA, in the following termed the 'beam spot', has an approximately Gaussian profile. For the data period studied in this paper the beam spot size is determined to be about $145 \mu \mathrm{m}$ in the horizontal and $25 \mu \mathrm{m}$ in the vertical direction. For each event the $e p$ collision point is determined in a primary vertex fit using the weighted average of the beam spot position, taking the above widths as errors, and position information from selected tracks in the event. The muon track under consideration is excluded from the fit. An average muon impact parameter resolution of $80 \mu \mathrm{m}$ is achieved, with comparable contributions from the muon track resolution and the uncertainty in the primary vertex position.

The transverse momentum $p_{t}^{\text {rel }}$ of the muon track (Figs. 4b and 5b) is calculated relative to the direction 

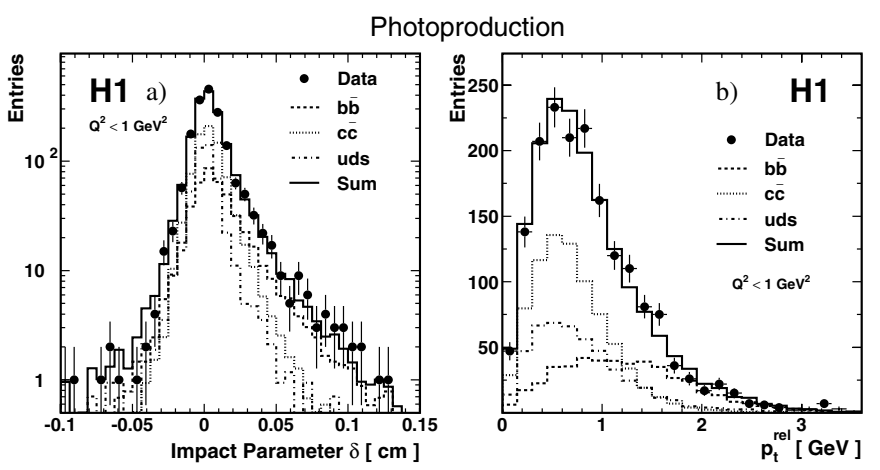

Fig. 4. Distributions in photoproduction of a the impact parameter $\delta$ of the muon track and $\mathbf{b}$ the transverse muon momentum $p_{t}^{\text {rel }}$ relative to the axis of the associated jet. Included in the figure are the estimated contributions of events arising from $b$ quarks (dashed line), $c$ quarks (dotted line) and the light quarks (dash-dotted line). The shapes of the distributions of the different sources are taken from the PYTHIA Monte Carlo simulation and their relative fractions are determined from a fit to the two-dimensional data distribution of $p_{t}^{\text {rel }}$ and $\delta$ (see text)

of the rest of the associated jet according to the formula

$$
p_{t}^{\mathrm{rel}}=\frac{\left|\boldsymbol{p}_{\boldsymbol{\mu}} \times\left(\boldsymbol{p}_{\text {jet }}-\boldsymbol{p}_{\boldsymbol{\mu}}\right)\right|}{\left|\boldsymbol{p}_{\text {jet }}-\boldsymbol{p}_{\boldsymbol{\mu}}\right|} .
$$

The quantities $\boldsymbol{p}_{\boldsymbol{\mu}}$ and $\boldsymbol{p}_{\text {jet }}$ are the momentum vectors of the muon and the jet in the laboratory frame, respectively.

The fraction of muons in the data that originate from beauty events is determined using a likelihood fit to the two-dimensional distribution of $\delta$ and $p_{t}^{\text {rel }}$ in the range $-0.1<\delta<0.1 \mathrm{~cm}$ and $0<p_{t}^{\text {rel }}<3.6 \mathrm{GeV}$. The combination of the two independent observables $\delta$ and $p_{t}^{\text {rel }}$ in the fit results in a significant improvement in the statistical precision of the measurement and a reduced sensitivity of the measured $b$ fraction to systematic uncertainties in the modelling of charm and light quark contributions. The shapes of the distributions $\delta$ and $p_{t}^{\text {rel }}$ for beauty ${ }^{2}$, charm and light quark events are obtained from the Monte Carlo simulation. In the fit, the relative fractions of the three components are adjusted such that the likelihood is maximised. The normalisation of the sum of the three components is fixed to match the data. The results of the fits are illustrated in Figs. 4 and 5. The contributions from beauty, charm and light quark events, with respective fractions of typically $30 \%, 50 \%$ and $20 \%$, are indicated. In all bins of the measurement, the data are well described by the sum of the three contributions. At large positive values of $\delta$ and at large values of $p_{t}^{\text {rel }}$, the beauty component (dashed line) becomes dominant.

Variations of the fit procedure are investigated and are found to give consistent results. For example, the $\delta$ and $p_{t}^{\text {rel }}$ distributions are fitted independently of each other. In another fit the relative contributions of charm and light quark events are fixed to the predictions of

\footnotetext{
${ }^{2}$ Both direct decays $b \rightarrow \mu X$ and indirect decays, e.g. $b \rightarrow$ $c X^{\prime} \rightarrow \mu X$, are taken into account.
}

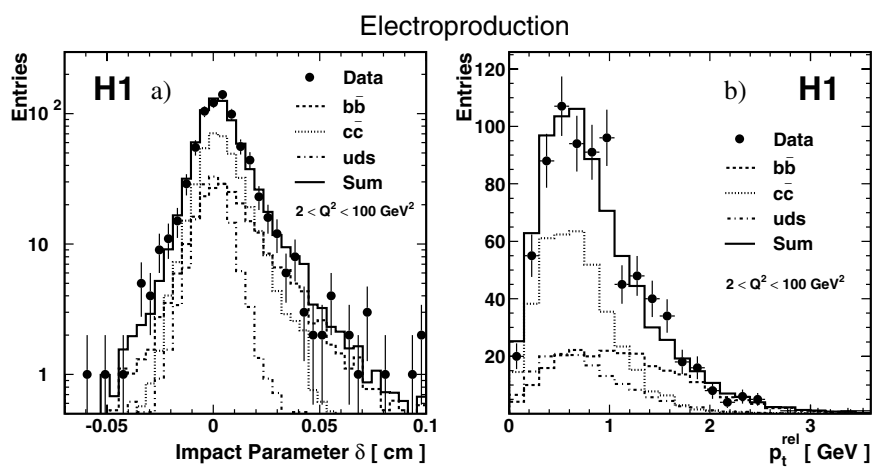

Fig. 5. Distributions in electroproduction of a the impact parameter $\delta$ of the muon track and $\mathbf{b}$ the transverse muon momentum $p_{t}^{\text {rel }}$ relative to the axis of the associated jet. Included in the figure are the estimated contributions of events arising from $b$ quarks (dashed line), $c$ quarks (dotted line) and the light quarks (dash-dotted line). The shapes of the distributions of the different sources are taken from the RAPGAP Monte Carlo simulation and their relative fractions are determined from a fit to the two-dimensional data distribution of $p_{t}^{\text {rel }}$ and $\delta$ (see text)
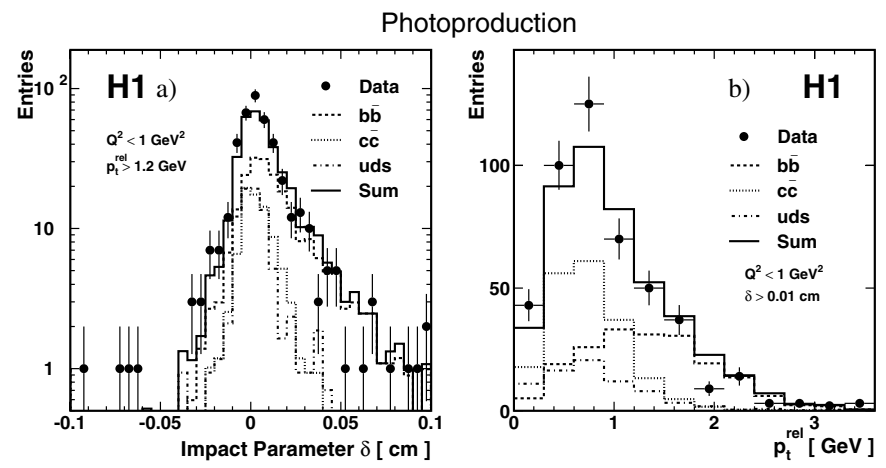

Fig. 6. Distributions in the restricted photoproduction sample of a the impact parameter $\delta$ for events with $p_{t}^{\text {rel }}>1.2 \mathrm{GeV}$ and $\mathbf{b}$ the transverse muon momentum $p_{t}^{\text {rel }}$ relative to the jet axis for tracks with impact parameter $\delta>0.01 \mathrm{~cm}$. The predictions for the contributions to the restricted sample from $b$ events (dashed line), $c$ events (dotted line) and light quark events (dash-dotted line), as determined from a fit to the twodimensional distribution of $p_{t}^{\text {rel }}$ and $\delta$ in the full data sample (see text), are also shown

the Monte Carlo simulation. Furthermore, the fits to the two-dimensional data distributions of $\delta$ and $p_{t}^{\text {rel }}$ are investigated in a beauty enriched subset of the $\delta$ and $p_{t}^{\text {rel }}$ phase space, as illustrated in the Figs. 6 and 7. Here, the distributions of the impact parameter $\delta$ (Figs. 6a and $7 \mathrm{a}$ ) and the relative transverse muon momentum $p_{t}^{\text {rel }}$ (Figs. 6b and 7b) are shown for the cuts $p_{t}^{\text {rel }}>1.2 \mathrm{GeV}$ and $\delta>0.01 \mathrm{~cm}$, respectively. The lines show the predictions for the different contributions in the restricted samples when using the results of the fits to the complete samples. The expected enhancement of the beauty contribution is observed and the quality of the description of the data illustrates the consistency between the results obtained using the two observables independently. 

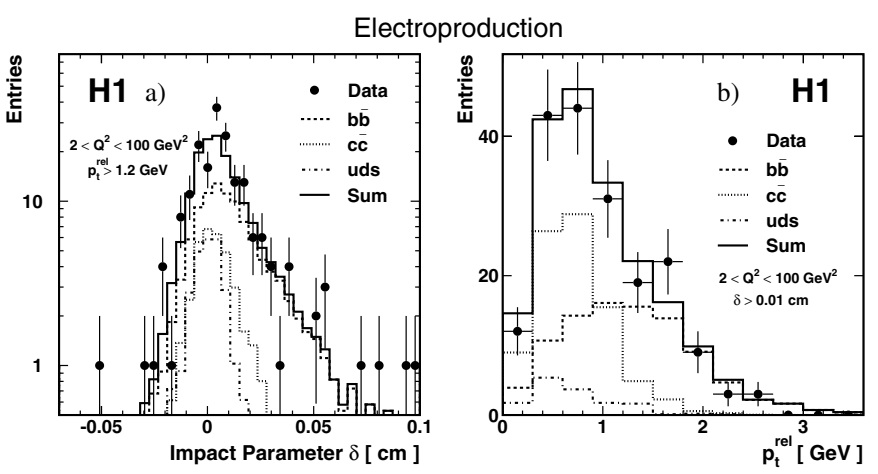

Fig. 7. Distributions in the restricted electroproduction sample of a the impact parameter $\delta$ for events with $p_{t}^{\text {rel }}>1.2 \mathrm{GeV}$ and $\mathbf{b}$ the transverse muon momentum $p_{t}^{\text {rel }}$ relative to the jet axis for tracks with impact parameter $\delta>0.01 \mathrm{~cm}$. The predictions for the contributions to the restricted sample from $b$ events (dashed line), $c$ events (dotted line) and light quark events (dash-dotted line), as determined from a fit to the twodimensional distribution of $p_{t}^{\text {rel }}$ and $\delta$ in the full data sample (see text), are also shown

In each kinematic bin of the measurement, the fit to the two-dimensional data distribution of $\delta$ and $p_{t}^{\text {rel }}$ is performed, using the data and Monte Carlo samples in that bin. The fitted number of muons coming from beauty events is translated into a cross section by correcting for detector efficiencies, acceptances and radiative effects and by dividing by the integrated luminosity.

\section{Systematic uncertainties}

Systematic uncertainties of the cross section measurement are evaluated by variations applied to the Monte Carlo simulations. The dominant errors come from the muon identification and muon track linking efficiencies, the modelling of the resolution of the muon impact parameter and the fragmentation models. The systematic errors assigned to the measured cross sections are listed in Table 3.

The muon track reconstruction efficiency in the CTD is known to a precision of about $2 \%$. An additional uncertainty of $2 \%$ comes from the requirement that two CST hits be associated with the central track, yielding a total uncertainty for the track reconstruction efficiency of $3 \%$. The uncertainty of the muon identification efficiency, including the reconstruction in the instrumented iron and the linking with the central track, is about $5 \%$.

The systematic error arising from the uncertainties of the CTD and CST track resolutions is estimated by varying the muon impact parameter resolution in the Monte Carlo simulations by $10 \%$. This leads to cross section changes of $7 \%$. To substantiate this result the following cross checks are performed and found to be consistent. The core and the tails of the distribution of the impact parameter resolution are varied separately. The description of the beam spot ellipse is tested by determining the cross sections separately for two independent samples with either more horizontal or more vertical muons. In addition, the muon impact parameter is calculated with respect to the average $e p$ collision point instead of the primary vertex.

The reconstruction of the direction of the jet associated to the muon is studied by varying the resolutions of the reconstructed jet directions in the Monte-Carlo simulations. The effect on the measured cross sections is about $2 \%$. The jet energy scale uncertainties are estimated by varying the LAr energy scale in the Monte Carlo simulations by $4 \%$. This leads to cross section changes of up to $4 \%$.

The trigger efficiencies are $70 \pm 3 \%$ for the photoproduction sample and $85 \pm 3 \%$ for the DIS sample, respectively. For the DIS sample, the uncertainty associated with reconstruction and identification of the scattered positron is estimated to be less than $2 \%$. The luminosity measurement contributes a global $1.5 \%$ error.

The dependence on the physics model used for the beauty signal and the charm background is studied using the CASCADE Monte Carlo generator instead of PYTHIA or RAPGAP, leading to cross section variations of about 5\%. Using the Lund [27] fragmentation model instead of the Peterson fragmentation function [26] causes changes in the measured cross sections of up to $7 \%$.

The modelling of the decays of the $b$-flavoured hadrons has been tested by varying the lifetimes and branching ratios of the different hadrons within the uncertainties of the world average values. The effects on the measured cross section are at the $2 \%$ level. Muons from $\pi^{ \pm}$or $K^{ \pm}$decays within the beam pipe and inside the sensitive volume of the CTD and CST exhibit a broad $\delta$ distribution. The contribution from these events in the light quark Monte Carlo simulation is varied by a factor of two, leading to cross section changes of $2 \%$.

The above systematic studies are performed separately for each bin of the cross section measurement. The systematic errors are found to be of similar size for all bins. For each bin a total systematic uncertainty of $14 \%$ is estimated by adding all contributions to the systematic error in quadrature.

\section{Results}

Differential beauty production cross sections are determined separately for the photoproduction and electroproduction samples. The results are listed in Tables $4-6$ and displayed in Figs. 8 to 11.

\subsection{Photoproduction measurement}

The visible range for the measurement for beauty photoproduction in dijet muon events is $Q^{2}<1 \mathrm{GeV}^{2}, 0.2<$ $y<0.8, p_{t}^{\mu}>2.5 \mathrm{GeV},-0.55<\eta^{\mu}<1.1, p_{t}^{\text {jet }_{1(2)}}>7(6)$ $\mathrm{GeV}$ and $\left|\eta^{\text {jet }}\right|<2.5$. For this range the total cross section is measured to be

$$
\begin{aligned}
\sigma_{\mathrm{vis}}\left(e p \rightarrow e b \bar{b} X \rightarrow e j j \mu X^{\prime}\right)= & 38.4 \pm 3.4 \text { (stat.) } \\
& \pm 5.4 \text { (sys.) pb. }
\end{aligned}
$$


Table 3. List of systematic uncertainties as discussed in Sect. 9. The total systematic error is obtained by adding all contributions in quadrature

\begin{tabular}{|c|c|c|}
\hline Source & $\begin{array}{c}\text { Photoproduction } \\
\Delta \sigma / \sigma[\%]\end{array}$ & $\begin{array}{c}\text { DIS } \\
\Delta \sigma / \sigma[\%]\end{array}$ \\
\hline \multicolumn{3}{|l|}{ Detector efficiencies } \\
\hline - Scattered positron & - & 2 \\
\hline - Trigger efficiency & 4 & 3 \\
\hline - Muon identification & 5 & 5 \\
\hline - CST+CTD tracks & 3 & 3 \\
\hline - Luminosity & 1.5 & 1.5 \\
\hline \multicolumn{3}{|l|}{ Track reconstruction } \\
\hline$-\delta$ resolution & 7 & 7 \\
\hline \multicolumn{3}{|l|}{ Jet reconstruction } \\
\hline - Jet axis & 2 & 2 \\
\hline - Hadronic energy scale & 4 & 4 \\
\hline \multicolumn{3}{|l|}{ MC model uncertainties: } \\
\hline - PYTHIA vs. CASCADE & 5 & - \\
\hline - RAPGAP vs. CASCADE & - & 5 \\
\hline - Fragmentation (Peterson vs. Lund) & 7 & 7 \\
\hline - Fragm. fractions, BRs, lifetimes & 2 & 2 \\
\hline$-K, \pi$ decays & 2 & 2 \\
\hline Total & 14 & 14 \\
\hline
\end{tabular}

Table 4. Differential cross sections for the process $e p \rightarrow e b \bar{b} X \rightarrow$ ejj $\mu X^{\prime}$ for the photoproduction sample in the kinematic range $Q^{2}<1 \mathrm{GeV}^{2}, 0.2<y<$ $0.8, p_{t}^{\mu}>2.5 \mathrm{GeV},-0.55<\eta^{\mu}<1.1, p_{t}^{\text {jet }_{1(2)}}>7(6) \mathrm{GeV}$ and $\eta^{\text {jet }}<2.5$

\begin{tabular}{|c|c|c|c|c|c|c|}
\hline \multirow{2}{*}{\multicolumn{2}{|c|}{$\eta^{\mu}$-range }} & \multirow[b]{2}{*}{$\eta^{\mu}$} & \multirow{3}{*}{$\begin{array}{l}\text { Measurement } \\
d \sigma / d \eta^{\mu} \\
{[\mathrm{pb}]}\end{array}$} & \multicolumn{3}{|c|}{ Experimental errors } \\
\hline & & & & stat. & syst. & total \\
\hline & & & & & & \\
\hline-0.55 & -0.15 & -0.35 & 19.1 & 3.4 & 2.7 & 4.3 \\
\hline-0.15 & 0.25 & 0.05 & 23.4 & 4.0 & 3.3 & 5.2 \\
\hline 0.25 & 0.65 & 0.45 & 23.9 & 3.9 & 3.3 & 5.1 \\
\hline 0.65 & 1.10 & 0.85 & 21.8 & 3.8 & 3.0 & 4.9 \\
\hline \multirow{2}{*}{\multicolumn{2}{|c|}{$\begin{array}{c}p_{t}^{\mu} \text {-range } \\
{[\mathrm{GeV}]}\end{array}$}} & $p_{t}^{\mu}$ & $d \sigma / d p_{t}^{\mu}$ & stat. & syst. & total \\
\hline & & {$[\mathrm{GeV}]$} & {$[\mathrm{pb} / \mathrm{GeV}]$} & {$[\mathrm{pb} / \mathrm{GeV}]$} & {$[\mathrm{pb} / \mathrm{GeV}]$} & {$[\mathrm{pb} / \mathrm{GeV}]$} \\
\hline 2.5 & 3.3 & 2.9 & 24.4 & 3.3 & 3.4 & 4.8 \\
\hline 3.3 & 5.0 & 4.1 & 8.1 & 1.1 & 1.1 & 1.6 \\
\hline 5.0 & 12.0 & 7.2 & 1.15 & 0.18 & 0.16 & 0.24 \\
\hline \multicolumn{2}{|c|}{$\begin{array}{c}p_{t}^{\text {jet }} \text {-range } \\
{[\mathrm{GeV}]}\end{array}$} & $\begin{array}{l}p_{t}^{\text {jet }} \\
{[\mathrm{GeV}]}\end{array}$ & $\begin{array}{l}d \sigma / d p_{t}^{\text {jet }} \\
{[\mathrm{pb} / \mathrm{GeV}]}\end{array}$ & $\begin{array}{l}\text { stat. } \\
{[\mathrm{pb} / \mathrm{GeV}]}\end{array}$ & $\begin{array}{l}\text { syst. } \\
{[\mathrm{pb} / \mathrm{GeV}]}\end{array}$ & $\begin{array}{l}\text { total } \\
{[\mathrm{pb} / \mathrm{GeV}]}\end{array}$ \\
\hline 7.0 & 10.0 & 8.8 & 6.3 & 0.8 & 0.9 & 1.2 \\
\hline 10.0 & 14.0 & 11.7 & 2.9 & 0.4 & 0.4 & 0.6 \\
\hline 14.0 & 25.0 & 18.3 & 0.83 & 0.14 & 0.12 & 0.18 \\
\hline \multicolumn{2}{|c|}{$x_{\gamma}^{\text {obs }}$-range } & $x_{\gamma}^{\text {obs }}$ & $\begin{array}{l}d \sigma / d x_{\gamma}^{\mathrm{obs}} \\
{[\mathrm{pb}]}\end{array}$ & $\begin{array}{l}\text { stat. } \\
{[\mathrm{pb}]}\end{array}$ & $\begin{array}{l}\text { syst. } \\
{[\mathrm{pb}]}\end{array}$ & $\begin{array}{l}\text { total } \\
{[\mathrm{pb}]}\end{array}$ \\
\hline 0.20 & 0.50 & 0.35 & 17.2 & 4.5 & 2.4 & 5.1 \\
\hline 0.50 & 0.75 & 0.63 & 21.4 & 5.2 & 3.0 & 6.0 \\
\hline 0.75 & 1.00 & 0.88 & 86.6 & 9.1 & 12.1 & 15.2 \\
\hline
\end{tabular}


Table 5. Differential cross sections for the process $e p \rightarrow e b \bar{b} X \rightarrow e j \mu X^{\prime}$ for the electroproduction sample in the kinematic range $2<Q^{2}<100 \mathrm{GeV}^{2}, 0.1<y<$ $0.7, p_{t}^{\mu}>2.5 \mathrm{GeV},-0.75<\eta^{\mu}<1.15, p_{t, \text { jet }}^{\text {Breit }}>6 \mathrm{GeV}$ and $\eta^{\text {jet }}<2.5$

\begin{tabular}{|c|c|c|c|c|c|c|}
\hline \multirow{2}{*}{\multicolumn{2}{|c|}{$\begin{array}{c}Q^{2} \text {-range } \\
{\left[\mathrm{GeV}^{2}\right]} \\
\end{array}$}} & \multirow{3}{*}{$\begin{array}{l}\begin{array}{l}Q^{2} \\
{\left[\mathrm{GeV}^{2}\right]}\end{array} \\
3.5\end{array}$} & \multirow{3}{*}{$\begin{array}{l}\text { Measurement } \\
d \sigma / d Q^{2} \\
{\left[\mathrm{pb} / \mathrm{GeV}^{2}\right]} \\
1.55\end{array}$} & \multicolumn{3}{|c|}{ Experimental errors } \\
\hline & & & & \multirow{2}{*}{$\begin{array}{l}\text { stat. } \\
{\left[\mathrm{pb} / \mathrm{GeV}^{2}\right]} \\
0.30\end{array}$} & \multirow{2}{*}{$\begin{array}{l}\text { syst. } \\
{\left[\mathrm{pb} / \mathrm{GeV}^{2}\right]} \\
0.22\end{array}$} & \multirow{2}{*}{$\begin{array}{l}\text { total } \\
{\left[\mathrm{pb} / \mathrm{GeV}^{2}\right.} \\
0.37\end{array}$} \\
\hline 2.0 & 5.0 & & & & & \\
\hline 5.0 & 18.0 & 9.5 & 0.297 & 0.075 & 0.042 & 0.086 \\
\hline 18.0 & 100.0 & 45.0 & 0.091 & 0.016 & 0.013 & 0.020 \\
\hline \multicolumn{2}{|c|}{$x$-range } & $\log x$ & $\begin{array}{l}d \sigma / d \log x \\
{[\mathrm{pb}]}\end{array}$ & $\begin{array}{l}\text { stat. } \\
{[\mathrm{pb}]}\end{array}$ & $\begin{array}{l}\text { syst. } \\
{[\mathrm{pb}]}\end{array}$ & $\begin{array}{l}\text { total } \\
{[\mathrm{pb}]}\end{array}$ \\
\hline-4.5 & -3.8 & -4.15 & 6.40 & 1.33 & 0.90 & 1.60 \\
\hline-3.8 & -3.1 & -3.45 & 9.72 & 1.71 & 1.36 & 2.18 \\
\hline-3.1 & -2.4 & -2.75 & 6.68 & 1.44 & 0.93 & 1.71 \\
\hline \multicolumn{2}{|c|}{$\eta^{\mu}$-range } & $\eta^{\mu}$ & $\begin{array}{l}d \sigma / d \eta^{\mu} \\
{[\mathrm{pb}]}\end{array}$ & $\begin{array}{l}\text { stat. } \\
{[\mathrm{pb}]}\end{array}$ & $\begin{array}{l}\text { syst. } \\
{[\mathrm{pb}]}\end{array}$ & $\begin{array}{l}\text { total } \\
{[\mathrm{pb}]}\end{array}$ \\
\hline-0.75 & -0.12 & -0.4 & 5.36 & 1.42 & 0.75 & 1.60 \\
\hline-0.12 & 0.50 & 0.2 & 7.40 & 1.64 & 1.03 & 1.94 \\
\hline 0.50 & 1.15 & 0.8 & 12.6 & 1.9 & 1.8 & 2.6 \\
\hline \multicolumn{2}{|c|}{$\begin{array}{c}p_{t}^{\mu} \text {-range } \\
{[\mathrm{GeV}]}\end{array}$} & $\begin{array}{l}p_{t}^{\mu} \\
{[\mathrm{GeV}]}\end{array}$ & $\begin{array}{l}d \sigma / d p_{t}^{\mu} \\
{[\mathrm{pb} / \mathrm{GeV}]}\end{array}$ & $\begin{array}{l}\text { stat. } \\
{[\mathrm{pb} / \mathrm{GeV}]}\end{array}$ & $\begin{array}{l}\text { syst. } \\
{[\mathrm{pb} / \mathrm{GeV}]}\end{array}$ & $\begin{array}{l}\text { total } \\
{[\mathrm{pb} / \mathrm{GeV}]}\end{array}$ \\
\hline 2.5 & 3.0 & 2.8 & 11.3 & 2.4 & 1.6 & 2.9 \\
\hline 3.0 & 3.8 & 3.4 & 8.05 & 1.39 & 1.13 & 1.79 \\
\hline 3.8 & 12.0 & 6.4 & 0.622 & 0.124 & 0.087 & 0.151 \\
\hline \multicolumn{2}{|c|}{$\begin{array}{c}p_{t, \mathrm{jet}}^{\text {Breit }} \text {-range } \\
{[\mathrm{GeV}]}\end{array}$} & $\begin{array}{l}p_{t, \text { jet }}^{\text {Breit }} \\
{[\mathrm{GeV}]}\end{array}$ & $\begin{array}{l}d \sigma / d p_{t, \text { jet }}^{\text {Breit }} \\
{[\mathrm{pb} / \mathrm{GeV}]}\end{array}$ & $\begin{array}{l}\text { stat. } \\
{[\mathrm{pb} / \mathrm{GeV}]}\end{array}$ & $\begin{array}{l}\text { syst. } \\
{[\mathrm{pb} / \mathrm{GeV}]}\end{array}$ & $\begin{array}{l}\text { total } \\
{[\mathrm{pb} / \mathrm{GeV}]}\end{array}$ \\
\hline 6.0 & 8.5 & 7.2 & 2.20 & 0.52 & 0.31 & 0.60 \\
\hline 8.5 & 12.0 & 10.0 & 1.96 & 0.31 & 0.27 & 0.42 \\
\hline 12.0 & 30.0 & 18.5 & 0.183 & 0.043 & 0.026 & 0.050 \\
\hline
\end{tabular}

Table 6. Measured cross sections with their statistical and systematic errors and corresponding predictions from NLO QCD calculations and Monte Carlo simulations in the kinematic range $Q^{2}<1 \mathrm{GeV}^{2}, 0.2<y<0.8, p_{t}^{\mu}>2.5 \mathrm{GeV}$, $-0.55<\eta^{\mu}<1.1, p_{t}^{\text {jet }_{1(2)}}>7(6) \mathrm{GeV}$ and $\eta^{\text {jet }}<2.5$ (photoproduction) and in the kinematic range $2<Q^{2}<100 \mathrm{GeV}^{2}$, $0.1<y<0.7, p_{t}^{\mu}>2.5 \mathrm{GeV},-0.75<\eta^{\mu}<1.15, p_{t, \text { jet }}^{\text {Breit }}>6$ $\mathrm{GeV}$ and $\left|\eta^{\text {jet }}\right|<2.5$ (electroproduction). The errors for the predictions from FMNR and HVQDIS give the systematic uncertainties as estimated from scale variations (see text)

\begin{tabular}{llll}
\hline \multicolumn{2}{c}{ Photoproduction } & \multicolumn{2}{c}{ Electroproduction } \\
$\sigma\left(e p \rightarrow e b \bar{b} X \rightarrow e j j \mu X^{\prime}\right)[\mathrm{pb}]$ & \multicolumn{2}{c}{$\sigma\left(e p \rightarrow e b \bar{b} X \rightarrow e j \mu X^{\prime}\right)[\mathrm{pb}]$} \\
\hline Data & $38.4 \pm 3.4 \pm 5.4$ & Data & $16.3 \pm 2.0 \pm 2.3$ \\
FMNR & $23.8_{-5.1}^{+7.4}$ & HVQDIS & $9.0_{-1.6}^{+2.6}$ \\
PYTHIA & 20.9 & RAPGAP & 6.3 \\
CASCADE & 22.6 & CASCADE & 9.8 \\
\hline
\end{tabular}

The NLO QCD calculation performed in the massive scheme with the FMNR program [9], as described in Sect. 6, yields for the same kinematic range a value of $23.8_{-5.1}^{+7.4} \mathrm{pb}$ which is 1.5 standard deviations below the data. The Monte Carlo programs PYTHIA and CAS-
CADE also predict a lower cross section than that measured in the data (see Table 6). The results of all three calculations are in good agreement with each other. An analysis using an independent H1 data sample was performed in [40] giving results consistent with this measurement.

Differential cross sections for beauty production are measured as a function of several kinematic variables, shown in Fig. 8 and listed in Table 4. The bins in which the measurement is made are identical to the bins in which the theory curves are presented. The measured cross sections are quoted at the point in the bin at which the binaveraged cross section equals the differential cross section, according to the Monte Carlo simulation. The data are compared with the expectations of the FMNR NLO QCD calculation and the PYTHIA and CASCADE generators.

The differential cross section measured as a function of the muon pseudo-rapidity $\eta^{\mu}$ (Fig. 8a) is flat in the phase space covered. The NLO QCD calculation describes the shape well. This is also true for both PYTHIA and CASCADE. The measurement agrees well with the values obtained by the ZEUS experiment [6] in their two central muon pseudo-rapidity bins, which cover a similar phase space. 


\section{Photoproduction}
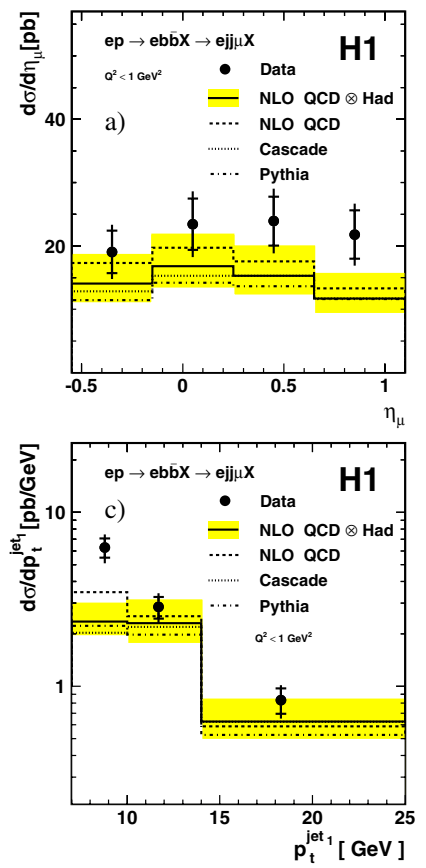
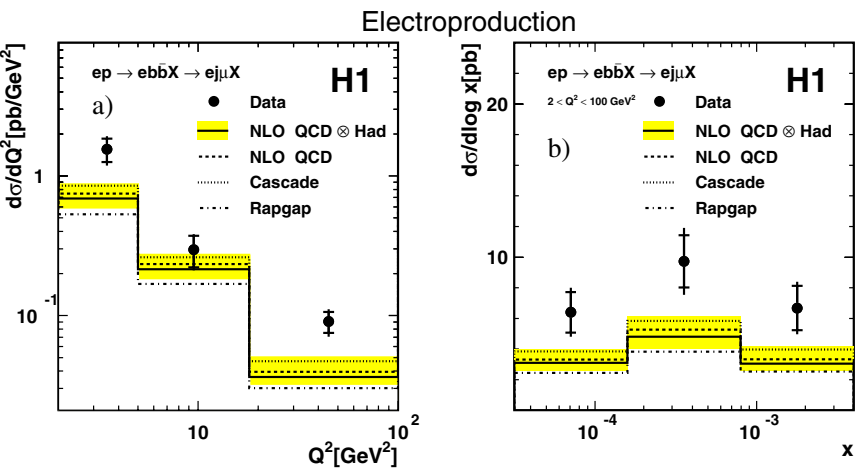

Fig. 9. Differential cross sections for the electroproduction process $e p \rightarrow e b \bar{b} X \rightarrow e j \mu X^{\prime}$ in the kinematic range $2<Q^{2}<$ $100 \mathrm{GeV}^{2}, 0.1<y<0.7, p_{t}^{\mu}>2.5 \mathrm{GeV},-0.75<\eta^{\mu}<1.15$, $p_{t, \text { jet }}^{\text {Breit }}>6 \mathrm{GeV}$ and $\left|\eta^{\text {jet }}\right|<2.5$. The cross sections are shown as functions of $\mathbf{a}$ the photon virtuality $Q^{2}$ and $\mathbf{b}$ the Bjorken scaling variable $x$. The inner error bars show the statistical error, the outer error bars represent the statistical and systematic uncertainties added in quadrature. NLO QCD predictions at the parton level (dashed line) are corrected to the hadron level (solid line) using the RAPGAP generator. The shaded band around the hadron level prediction indicates the systematic uncertainty as estimated from scale variations (see text). Predictions from the Monte Carlo generator programs CASCADE (dotted line) and RAPGAP (dash-dotted line) are also shown

cess $e p \rightarrow e b \bar{b} X \rightarrow$ ejj $\mu X^{\prime}$ in the kinematic range $Q^{2}<$ $\mathrm{GeV}^{2}, 0.2<y<0.8, p_{t}^{\mu}>2.5 \mathrm{GeV},-0.55<\eta^{\mu}<1.1$, $p_{t}^{\text {jet }_{1(2)}}>7(6) \mathrm{GeV}$ and $\left|\eta^{\text {jet }}\right|<2.5$. The cross sections are shown as functions of $\mathbf{a}$ the muon pseudo-rapidity $\eta^{\mu}$, $\mathbf{b}$ the muon transverse momentum $p_{t}^{\mu}$, $\mathbf{c}$ the jet transverse momentum $p_{t}^{\text {jet }_{1}}$ of the highest transverse momentum jet and $\mathbf{d}$ the quantity $x_{\gamma}^{\text {obs }}$. The inner error bars show the statistical error, the outer error bars represent the statistical and systematic uncertainties added in quadrature. The NLO QCD predictions at the parton level (dashed line) are corrected to the hadron level (solid line) using the PYTHIA generator. The shaded band around the hadron level prediction indicates the systematic uncertainties as estimated from scale variations (see text). Predictions from the Monte Carlo generator programs CASCADE (dotted line) and PYTHIA (dash-dotted line) are also shown

The differential cross sections measured as a function of the muon transverse momentum $p_{t}^{\mu}$ and of the transverse momentum of the leading jet $p_{t}^{\text {jet }}$ (Fig. 8b and c) fall steeply with increasing transverse momentum. The NLO calculation clearly predicts a less steep behaviour and is lower than the data in the lowest momentum bin by roughly a factor of 2.5. At higher transverse momenta better agreement is observed. Similar conclusions can be drawn for both the PYTHIA and CASCADE predictions, although the latter predicts a slightly harder $p_{t}^{\text {jet }}$ spectrum than the other calculations.

Figure $8 \mathrm{~d}$ shows the differential cross sections as a function of $x_{\gamma}^{\text {obs }}$. A significant fraction of the data is found at $x_{\gamma}^{\text {obs }}<0.75$, i.e. in the region in which resolved photon processes (Figs. 1b to d) are enhanced. In this observable the NLO calculation suffers from large uncertainties due to the scale variations. Furthermore, the parton to

hadron level corrections are large due to the fact that a single parton can produce more than one jet at the hadron level leading to migrations in $x_{\gamma}^{\text {obs }}$. Within the large uncertainties, the NLO calculation describes the $x_{\gamma}^{\text {obs }}$ differential cross sections reasonably well. The PYTHIA simulation includes a 35\% contribution from resolved photon processes, which are dominated by flavour excitation processes such as those shown in Figs. 1c and d. Due to the large fraction of resolved photon processes, PYTHIA predicts a relatively high cross section value in the lowest $x_{\gamma}^{\text {obs }}$ bin (Fig. 8d), which matches the data quite well. However, in the largest bin, $x_{\gamma}^{\text {obs }}>0.75$, the PYTHIA prediction is too low. In contrast, CASCADE succeeds in describing the cross section of the data at large values of $x_{\gamma}^{\text {obs }}$ while it is too low at smaller values of $x_{\gamma}^{\text {obs }}$.

The results of this analysis are compared with the previous $\mathrm{H} 1$ measurement [1] in which somewhat softer jet and muon cuts than in this analysis were used for the event selection. The measured cross section for the process $e p \rightarrow e b \bar{b} X \rightarrow e j j \mu X^{\prime}$ is extrapolated to the inclusive $b$ quark cross section, ep $\rightarrow b \bar{b} X \rightarrow \mu X^{\prime}$, in the kinematic region $Q^{2}<1 \mathrm{GeV}^{2}, 0.1<y<0.8, p_{t}^{\mu}>2 \mathrm{GeV}$ and $35^{\circ}<\theta^{\mu}<130^{\circ}$, as in [1]. The extrapolation is performed using the Monte Carlo program AROMA[41] which was also used in [1]. The result, scaled to $820 \mathrm{GeV}$ proton beam energy, is $107.3 \pm 9.5$ (stat.) \pm 15.1 (sys.) pb, which is 2.3 standard deviations lower than the value of $176 \pm 16$ (stat.) ${ }_{-17}^{+26}($ sys.) pb obtained in [1]. 
Electroproduction
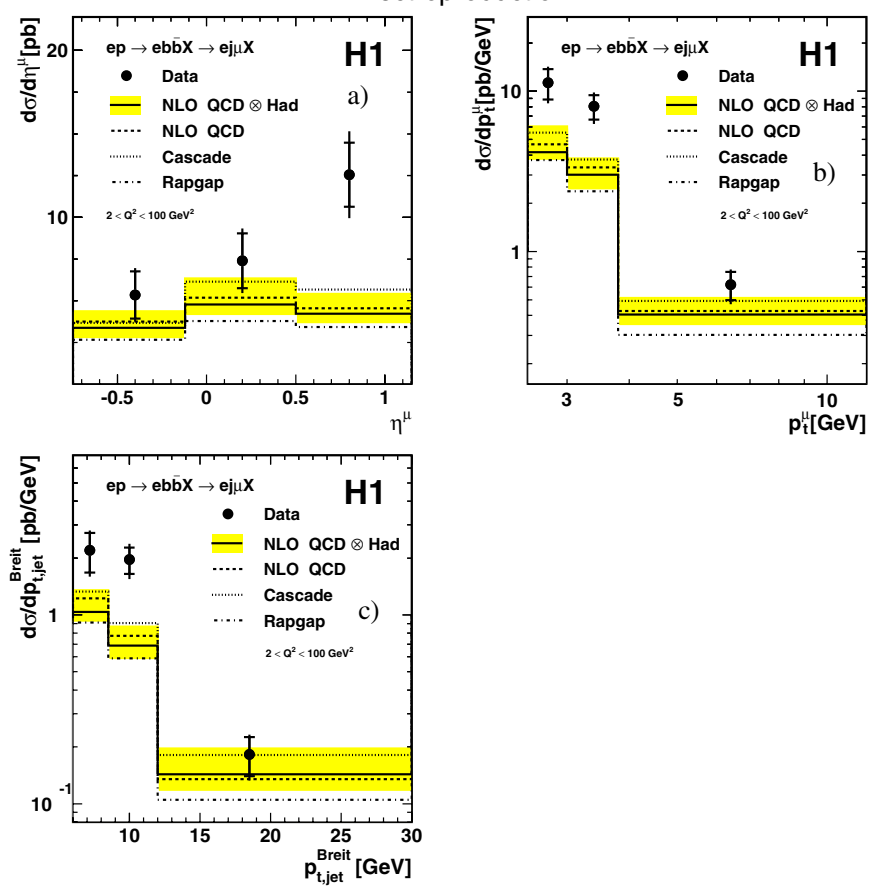

Fig. 10. Differential cross sections for the electroproduction process $e p \rightarrow e b \bar{b} X \rightarrow e j \mu X^{\prime}$ in the kinematic range $2<Q^{2}<$ $100 \mathrm{GeV}^{2}, 0.1<y<0.7, p_{t}^{\mu}>2.5 \mathrm{GeV},-0.75<\eta^{\mu}<1.15$ $p_{t, \text { jet }}^{\text {Breit }}>6 \mathrm{GeV}$ and $\left|\eta^{\text {jet }}\right|<2.5$. The cross sections are shown as functions of a the muon pseudo-rapidity $\eta^{\mu}$, $\mathbf{b}$ the muon transverse momentum $p_{t}^{\mu}$ and $\mathbf{c}$ the transverse momentum $p_{t, \text { jet }}^{\text {Breit }}$ of the leading jet in the Breit frame. The inner error bars show the statistical error, the outer error bars represent the statistical and systematic uncertainties added in quadrature. The NLO QCD predictions at the parton level (dashed line) are corrected to the hadron level (solid line) using the RAPGAP generator. The shaded band around the hadron level prediction indicates the systematic uncertainty as estimated from scale variations (see text). Predictions from the Monte Carlo generator programs CASCADE (dotted line) and RAPGAP (dash-dotted line) are also shown

\subsection{Electroproduction measurement}

The beauty electroproduction cross section is measured in the visible range $2<Q^{2}<100 \mathrm{GeV}^{2}, 0.1<y<0.7$, $p_{t}^{\mu}>2.5 \mathrm{GeV},-0.75<\eta^{\mu}<1.15, p_{t, \text { jet }}^{\text {Breit }}>6 \mathrm{GeV}$ and $\left|\eta^{\text {jet }}\right|<2.5$, yielding

$$
\begin{aligned}
\sigma_{\mathrm{vis}}\left(e p \rightarrow e b \bar{b} X \rightarrow e j \mu X^{\prime}\right)= & 16.3 \pm 2.0 \text { (stat.) } \\
& \pm 2.3 \text { (sys.) pb. }
\end{aligned}
$$

The prediction of the NLO QCD calculation in the massive scheme using the program HVQDIS is $9.0_{-1.6}^{+2.6} \mathrm{pb}$, which is 1.8 standard deviations below the data. The Monte Carlo programs RAPGAP and CASCADE also predict a lower cross section than that measured in the data (see Table 6).

Differential cross section measurements are presented in Figs. 9 and 10 and in Table 5. The data are compared with the expectations of the HVQDIS NLO QCD calculation and the RAPGAP and CASCADE generators. The differential cross section as a function of the photon virtuality $Q^{2}$ is shown in Fig. 9a. The NLO calculation describes the shape well, but lies below the data. The prediction of CASCADE is similar to that of the NLO calculation while RAPGAP, which only contains the direct photon contribution to the cross section, is somewhat further below the data. The differential cross section as a function of the scaling variable $x$ is shown in Fig. 9b. The various calculations also describe the shape of the data well, while the overall normalisation is again too low.

In Fig. 10, the differential cross sections are presented as functions of the muon and leading jet kinematics. The differential cross section measured in bins of the muon pseudo-rapidity $\eta^{\mu}$ (Fig. 10a) exhibits a rise towards the forward region, which is not reproduced by the NLO and Monte Carlo calculations. The differential cross sections measured as a function of the transverse momenta of the muon $p_{t}^{\mu}$ and of the jet in the Breit frame $p_{t, \text { jet }}^{\text {Breit }}$ (Figs. 10b and c) show a steep distribution, as is the case in photoproduction (Figs. $8 \mathrm{~b}$ and $\mathrm{c}$ ). The shapes of the NLO QCD, RAPGAP and CASCADE predictions are all very similar. As in photoproduction, the measured electroproduction cross sections as a function of the muon and jet transverse momenta show a steeper behaviour than the predictions of the NLO calculations and the Monte Carlo simulations and significantly exceed the predictions in the lowest bins.

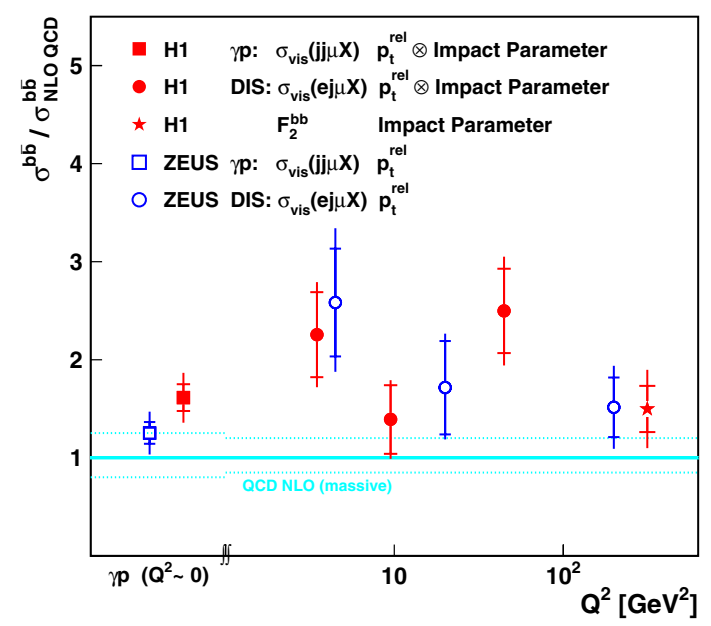

Fig. 11. Ratio of beauty production cross section measurements at HERA to NLO QCD predictions. The results of this paper (solid circles and squares) are compared with ratios determined using the measurements taken from $[6,7,5]$. The photoproduction points are plotted at different horizontal positions for better visibility. Note that cross section definitions and kinematic ranges are somewhat different for the different data points. The dotted lines indicate the typical theoretical error due to scale uncertainties. The theoretical prediction used to form the ratio with the measurement by the ZEUS Experiment [6], shown as an open square, is calculated using the same program and parameter choices as for the prediction for this measurement (full square). Different parameter choices, e.g. for the modelling of the hadronisation and decay of the $B$-hadron, lead to a variation of the prediction of $\sim 10 \%$ 
Figure 11 presents a summary of recent HERA beauty cross section measurements as a function of the photon virtuality $Q^{2}$. The figure shows the ratios of the measured cross sections [5-7] and the corresponding next-to-leading order predictions where FMNR is used for the photoproduction and HVQDIS for the DIS region. The dotted lines indicate the typical theory error due to scale uncertainties. General agreement is seen between the results from H1 and ZEUS, the data tending to be somewhat above the NLO predictions.

\section{Conclusions}

Differential beauty production cross sections are measured in $e p$ collisions at HERA both in photoproduction $\left(Q^{2}<\right.$ $\left.1 \mathrm{GeV}^{2}\right)$ and in electroproduction $\left(2<Q^{2}<100 \mathrm{GeV}^{2}\right)$. The event selection requires the presence of at least one jet (two jets) in the DIS (photoproduction) sample and a muon in the central pseudo-rapidity range. For the first time at HERA, beauty events are identified using both the transverse momentum of the muon relative to the jet axis and the large impact parameter of the muon. The cross sections presented here are in general agreement with those obtained by the ZEUS experiment. The data are compared with predictions based on NLO QCD calculations in the massive scheme and with the expectations of Monte Carlo generators which use leading order matrix elements and parton showers. The predictions from all these calculations are similar in both normalisation and shape.

In both photoproduction and DIS, the total cross section measurements are somewhat higher than the predictions. The excess is observed mainly at small muon and jet transverse momenta, while at larger momenta a reasonable description is obtained. In photoproduction a significant contribution to the cross section is observed in the region of small values of the observable $x_{\gamma}^{\text {obs }}$, where contributions from resolved photon events are enhanced. In this region the best description of the data is given by the PYTHIA simulation, which incorporates flavour excitation processes in which the beauty quark is a constituent of the resolved photon or the proton. In DIS, the observed excess is pronounced at large muon pseudo-rapidities. The shape of the cross section as function of the photon virtuality $Q^{2}$ is reproduced by the QCD calculations over the full range covered by the measurement presented in this paper, from quasi-real photons up to virtualities of about $4 m_{b}^{2}$.

Acknowledgements. We are grateful to the HERA machine group whose outstanding efforts have made this experiment possible. We thank the engineers and technicians for their work in constructing and maintaining the $\mathrm{H} 1$ detector, our funding agencies for financial support, the DESY technical staff for continual assistance and the DESY directorate for support and for the hospitality which they extend to the non-DESY members of the collaboration.

\section{References}

1. C. Adloff et al. [H1 Collaboration], Phys. Lett. B 467, 156 (1999) [hep-ex/9909029]; [Erratum-ibid. B 518, 331 (2001)]

2. J. Breitweg et al. [ZEUS Collaboration], Eur. Phys. J. C 18, 625 (2001) [hep-ex/0011081]

3. F. Abe et al. [CDF Collaboration], Phys. Rev. Lett. 71, 2396 (1993); Phys. Rev. D 53, 1051 (1996) [hepex/9508017]; S. Abachi et al. [D0 Collaboration], Phys. Rev. Lett. 74, 3548 (1995); Phys. Lett. B 370, 239 (1996)

4. M. Acciarri et al. [L3 Collaboration], Phys. Lett. B 503, 10 (2001) [hep-ex/0011070]

5. A. Aktas et al. [H1 Collaboration], to appear in Eur. Phys. J. C., [hep-ex/0411046]

6. S. Chekanov et al. [ZEUS Collaboration], Phys. Rev. D 70, 012008 (2004) [hep-ex/0312057]

7. S. Chekanov et al. [ZEUS Collaboration], Phys. Lett. B 599, 173 (2004) [hep-ex/0405069]

8. D. Pitzl et al., Nucl. Instrum. Meth. A 454, 334 (2000) [hep-ex/0002044]

9. S. Frixione, P. Nason, G. Ridolfi, Nucl. Phys. B 454, 3 (1995) [hep-ph/9506226]

10. B.W. Harris, J. Smith, Nucl. Phys. B 452, 109 (1995)

11. B.A. Kniehl, M. Krämer, G. Kramer, M. Spira, Phys. Lett. B 356, 539 (1995) [hep-ph/9505410]; B.A. Kniehl, G. Kramer, M. Spira, Z. Phys. C 76, 689 (1997) [hepph/9610267]; J. Binnewies, B.A. Kniehl, G. Kramer, Z. Phys. C 76, 677 (1997) [hep-ph/9702408]; J. Binnewies, B.A. Kniehl, G. Kramer, Phys. Rev. D 58, 014014 (1998) [hep-ph/9712482]; M. Cacciari, M. Greco, Phys. Rev. D 55, 7134 (1997) [hep-ph/9702389]

12. T. Sjöstrand, Comput. Phys. Commun. 39, 347 (1986); T. Sjöstrand, M. Bengtsson, Comput. Phys. Commun. 43, 367 (1987); T. Sjöstrand, Comput. Phys. Commun. 82, 74 (1994); T. Sjöstrand et al. , Comput. Phys. Commun. 135, 238 (2001) [hep-ph/0010017]

13. H. Jung, Comput. Phys. Commun. 86, 147 (1995)

14. H. Jung, G.P. Salam, Eur. Phys. J. C 19, 351 (2001) [hepph/0012143]; H. Jung, Comput. Phys. Commun. 143, 100 (2002) [hep-ph/0109102]

15. V.N. Gribov, L.N. Lipatov, Yad. Fiz. 15, 781 (1972) [Sov. J. Nucl. Phys. 15, 438 (1972)]; G. Altarelli, G. Parisi, Nucl. Phys. B 126, 298 (1977); Y.L. Dokshitzer, Sov. Phys. JETP 46, 641 (1977) [Zh. Eksp. Teor. Fiz. 73, 1216] (1977)

16. M. Ciafaloni, Nucl. Phys. B 296, 49 (1988); S. Catani, F. Fiorani, G. Marchesini, Phys. Lett. B 234, 339 (1990); S. Catani, F. Fiorani, G. Marchesini, Nucl. Phys. B 336, 18 (1990); G. Marchesini, Nucl. Phys. B 445, 49 (1995)

17. I. Abt et al. [H1 Collaboration], Nucl. Instrum. Meth. A 386, 310 (1997) and 348

18. R.D. Appuhn et al. [H1 SPACAL Group], Nucl. Instrum. Meth. A 386, 397 (1997)

19. S.D. Ellis, D.E. Soper, Phys. Rev. D 48, 3160 (1993) [hep$\mathrm{ph} / 9305266]$

20. S. Catani, Y.L. Dokshitzer, M.H. Seymour, B.R. Webber, Nucl. Phys. B 406, 187 (1993)

21. J.M. Butterworth, J.P. Couchman, B.E. Cox, B.M. Waugh, Comput. Phys. Commun. 153, 85 (2003) [hep-ph/0210022]

22. C. Adloff et al. [H1 Collaboration], Z. Phys. C 74, 221 (1997) [hep-ex/9702003] 
23. A. Blondel, F. Jacquet, in: U. Amaldi (ed.) Proc. Study of an ep Facility for Europe, DESY 79/48, 391 (1979)

24. U. Bassler, G. Bernardi, Nucl. Instrum. Meth. A 361, 197 (1995) [hep-ex/9412004]

25. R.P. Feynman, Photon-Hadron Interactions, Benjamin N.Y. (1972)

26. C. Peterson, D. Schlatter, I. Schmitt, P.M. Zerwas, Phys. Rev. D 27, 105 (1983)

27. B. Andersson, G. Gustafson, G. Ingelman, T. Sjöstrand, Phys. Rept. 97, 31 (1983)

28. R. Brun et al. , CERN-DD/EE-84-1 (1987)

29. A. Kwiatkowski, H. Spiesberger, H.J. Möhring, Comput. Phys. Commun. 69, 155 (1992)

30. H. Jung, Mod. Phys. Lett. A 19, 1 (2004) [hep$\mathrm{ph} / 0311249]$

31. J.R. Andersen et al. [Small x Collaboration], Eur. Phys. J. C 35, 67 (2004) [hep-ph/0312333]

32. M. Hansson, H. Jung, hep-ph/0309009

33. H.L. Lai et al. [CTEQ Collaboration], Eur. Phys. J. C 12, 375 (2000) [hep-ph/9903282]
34. M. Glück, E. Reya, A. Vogt, Phys. Rev. D 45, 3986 (1992); M. Glück, E. Reya, A. Vogt, Phys. Rev. D 46, 1973 (1992)

35. K. Hagiwara et al. [Particle Data Group], Phys. Rev. D 66, 010001 (2002)

36. K. Abe et al. [Belle Collaboration], Phys. Lett. B 547, 181 (2002) [hep-ex/0208033]

37. B. Aubert et al. [BABAR Collaboration], Phys. Rev. D 67, 031101 (2003) [hep-ex/0208018]

38. P. Nason, C. Oleari, Nucl. Phys. B 565, 245 (2000) [hep$\mathrm{ph} / 9903541]$

39. A.D. Martin, W.J. Stirling, R.G. Roberts, Phys. Lett. B 354, 155 (1995) [hep-ph/9502336]; A.D. Martin, R.G. Roberts, W.J. Stirling, R.S. Thorne, Eur. Phys. J. C 4, 463 (1998) [hep-ph/9803445]

40. J. Kroseberg, Dissertation, Univ. Zürich, 2002, unpublished, available at http://www-h1.desy.de/publications/theses.list.html

41. G. Ingelman, J. Rathsman, G.A. Schuler, Comput. Phys. Commun. 101, 135 (1997) [hep-ph/9605285] 